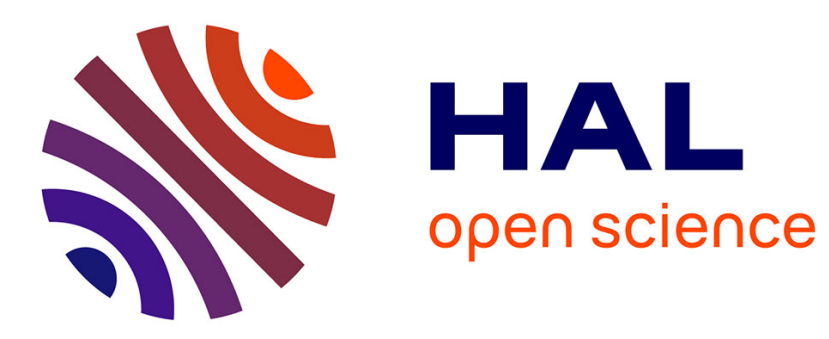

\title{
Stochastic receding horizon control with output feedback and bounded controls
}

Peter Hokayem, Eugenio Cinquemani, Debasish Chatterjee, Federico Ramponi, John Lygeros

\section{To cite this version:}

Peter Hokayem, Eugenio Cinquemani, Debasish Chatterjee, Federico Ramponi, John Lygeros. Stochastic receding horizon control with output feedback and bounded controls. Automatica, 2012, 48 (1), pp.77-88. 10.1016/j.automatica.2011.09.048 . hal-00762600

\section{HAL Id: hal-00762600 \\ https://inria.hal.science/hal-00762600}

Submitted on 7 Dec 2012

HAL is a multi-disciplinary open access archive for the deposit and dissemination of scientific research documents, whether they are published or not. The documents may come from teaching and research institutions in France or abroad, or from public or private research centers.
L'archive ouverte pluridisciplinaire HAL, est destinée au dépôt et à la diffusion de documents scientifiques de niveau recherche, publiés ou non, émanant des établissements d'enseignement et de recherche français ou étrangers, des laboratoires publics ou privés. 


\title{
Stochastic receding horizon control with output feedback and bounded controls
}

\author{
Peter Hokayem $^{\text {a }}$, Eugenio Cinquemani ${ }^{\mathrm{b}}$, Debasish Chatterjee ${ }^{\mathrm{a}}$, \\ Federico Ramponi ${ }^{\text {a }}$, John Lygeros ${ }^{\mathrm{a}}$ \\ a Automatic Control Laboratory, ETH Zürich, Physikstrasse 3, 8092 Zürich, Switzerland \\ ' INRIA Grenoble-Rhône-Alpes, 655 avenue de l'Europe, Montbonnot, 38334 Saint Ismier Cedex, France
}

\begin{abstract}
We study the problem of receding horizon control for stochastic discrete-time systems with bounded control inputs and incomplete state information. Given a suitable choice of causal control policies, we first present a slight extension of the Kalman filter to estimate the state optimally in mean-square sense. We then show how to augment the underlying optimization problem with a negative drift-like constraint, yielding a second-order cone program to be solved periodically online. We prove that the receding horizon implementation of the resulting control policies renders the state of the overall system mean-square bounded under mild assumptions. We also discuss how some quantities required by the finite-horizon optimization problem can be computed off-line, thus reducing the on-line computation.
\end{abstract}

Key words: Predictive control, output feedback, constrained control, state estimation, stochastic control

\section{Introduction}

A considerable amount of research has been devoted to deterministic receding horizon control, see, for example, $[35,32]$ and references therein. This resulted in proofs of recursive feasibility and stability of receding horizon control laws in the noise-free deterministic setting. These techniques can be extended to the robust case, i.e., whenever there is exogenous noise or parametric uncertainty of bounded nature entering the system. The counterpart for stochastic systems subject to process noise, imperfect state measurements, and bounded control inputs, however, is still lacking. The principal obstacle is posed by the fact that it may not be possible to determine an a priori bound on the support of the noise, for example, whenever the noise is additive and Gaussian. This extra ingredient complicates both the stability and the feasibility proofs: the noise, at least in the additive case, eventually drives the state outside any bounded set no

\footnotetext{
* This paper was not presented at any IFAC meeting. Corresponding author P. Hokayem. Tel. +41 44632 0403. Fax +41446321211.

Email addresses: hokayem@control.ee.ethz.ch (Peter Hokayem), eugenio.cinquemani@inria.fr (Eugenio Cinquemani), chatterjee@control.ee.ethz.ch (Debasish Chatterjee), ramponif@control.ee.ethz.ch (Federico Ramponi), lygeros@control.ee.ethz.ch (John Lygeros).
}

matter how large the latter is taken to be, and employing any standard linear state feedback means that any hard bounds on the control inputs will eventually be violated.

In this article we propose a solution to the general receding horizon control problem for linear systems with noisy process dynamics, imperfect state information, and bounded control inputs. Both the process and measurement noise sequences are assumed to enter the system in an additive fashion, and we require that the designed control policies satisfy hard bounds. Periodically at times $t=0, N_{c}, 2 N_{c}, \cdots$, where $N_{c}$ is the control horizon, a certain finite-horizon optimal control problem is solved over a prediction (or optimization) horizon $N \geqslant N_{c}$. The cost to be minimized is the standard expectation of the sum of cost-per-stage functions that are quadratic in the state and control inputs $[7,8]$. We can also include at the design level some variance-like bounds on the predicted future states and inputs - this is one possible way to impose soft state constraints that are in spirit similar to integrated chance-constraints, e.g., in [26,27].

There are several key challenges inherent to our setup. First, since the state information is imperfect one needs a filter to estimate the state. Second, in the presence of unbounded (e.g., Gaussian) noise, it is not possible in general to ensure any bound on the control values gener- 
ated via linear state feedback; the additive nature of the noise ensures that the state exits from any fixed bounded set at some time almost surely, implying the necessity of nonlinear feedback policies. This issue is further complicated by the fact that only incomplete state information is available. Third, it is unclear whether the application of the bounded control policies stabilizes the system in any reasonable sense. For a deterministic discrete-time linear system, $x_{t+1}=A x_{t}+B u_{t}$, it is not possible to globally asymptotically stabilize the system, if the matrix $A$ has unstable eigenvalues, see, for example, [46] and references therein. Moreover, in the presence of stochastic process noise the hope for achieving asymptotic stability is obviously not realistic. In this article we relax the notion of stability to mean-square boundedness of the state and impose the extra conditions that the system matrix $A$ is Lyapunov (or neutrally) stable and that the pair $(A, B)$ is stabilizable.

The main contributions of this paper can be summarized as follows: Given a suitable subclass of bounded causal feedback policies, we show how to augment the finite-horizon optimal control problem to be solved periodically every $N_{c}$ steps with a stability constraint and that the resulting optimization problem can be approximated to a globally feasible second-order cone program (SOCP). Under the assumption that the process and measurement noise are Gaussian, (even though the bounded inputs requirement makes the problem inherently nonlinear and the process statistics are nonGaussian, ) it turns out that Kalman filtering techniques can indeed be utilized. We rely on a low-complexity algorithm (essentially similar to standard Kalman filtering) for updating the conditional density of the state, given the history of the previous outputs, and report tractable solutions for the off-line computation of the time-dependent variance and covariance matrices in the optimization program. Finally, we show that the recursive application of the resulting control policies renders the state of the overall system mean-square bounded. This article builds upon and generalizes the earlier stability results in $[39,21]$ that were derived for the perfect state information case. Also, the current results generalize those in $[20,16]$ from the perfect state information case to the imperfect state information case. In particular, if we have full state information available, the control policy proposed in this article reduces to that proposed in [20] and to a special case of the policies proposed in [16] where the vector space is spanned by a single function. However, none of the earlier results, including those in [22], are able to deal with recursive feasibility and stability for the setup of this article. Moreover, the control policy structure in this article is different from that in [22].

Related Work The research on stochastic receding horizon control is broadly subdivided into two parallel lines: the first treats multiplicative noise that enters the state equations, and the second caters to additive noise. The case of multiplicative noise has been treated in $[38,14,18]$. In [38], the noise enters the state equation multiplicatively and mixed hard state-input constraints are relaxed into expectation constraints. Terminal constraints are imposed as well that render the overall MPC scheme stable under full state feedback. The authors in [18] treat the case of uncertain output measurement matrix $(C)$ and solve the MPC problem under probabilistic constraints on the outputs and full state feedback. In [14] the stochastic MPC problem is treated under full state feedback and multiplicative noise entering the state equation. The proposed scheme comprises a pre-stabilizing linear state feedback control part and an open-loop part. The pre-stabilizing feedback gain is computed off-line and only the open-loop part is optimized online. The results in [15] extend those in [14] to the case of additive noise as well. However, both results [15] and [14] involve a pre-stabilizing state feedback controller and hence no hard input bounds can be imposed.

We focus in this article on the additive noise case. The approach proposed here stems from and generalizes the idea of affine parametrization of control policies for finite-horizon linear quadratic problems proposed in $[3,2]$, utilized within the robust MPC framework in $[2,30,19]$ for full state feedback, and in [43] for output feedback with Gaussian state and measurement noise inputs. More recently, this affine approximation was utilized in [41] for both the robust deterministic and the stochastic setups in the absence of control bounds, and optimality of affine policies in the scalar deterministic case was reported in [10]. In [9] the authors reformulate the stochastic programming problem as a deterministic one with bounded noise support and solve a robust optimization problem over a finite horizon, followed by estimating the performance when the noise can take unbounded values, i.e., when the noise is unbounded, but takes high values with low probability. A similar approach was utilized in [36] as well. There are also other approaches, e.g., those employing randomized algorithms as in $[1,11,33]$. Results on obtaining lower bounds on the value functions of the stochastic optimization problem have been recently reported in [44], and a novel stochastic MPC scheme based on the scenario approach has appeared in [5]. Other works employing probabilistic constraints may be found in [40,29]. In [34], an inputto-state stability approach is employed and stability is shown under full state feedback and bounded additive process noise. An MPC scheme for systems with imperfect state information has been proposed in [45] under general hypotheses with probabilistic constraints. However, the ability to deal with noise of an unbounded nature (for example Gaussian) is still absent, in which stability and recursive feasibility could not be proven in [45] under bounded control inputs.

The rest of this article is organized as follows. We formulate the stochastic receding horizon control problem with all the underlying assumptions, the construction of the estimator, and the main optimization problem to 
be solved in Section 2. We provide the main results pertaining to tractability of the optimization problem and mean-square boundedness of the closed-loop system in Section 3. We comment on the obtained results and provide some extensions in Section 4 . We then present numerical examples in Section 5 and conclude in Section 6 . Finally, we provide the proofs in the Appendix.

Notation Let $(\Omega, \mathfrak{F}, \mathbb{P})$ be a general probability space. We denote the conditional expectation given the sub- $\sigma$ algebra $\mathfrak{F}^{\prime}$ of $\mathfrak{F}$ as $\mathbb{E}_{\mathfrak{F}^{\prime}}[$.$] . For any random vector s$ we let $\Sigma_{s}:=\mathbb{E}\left[s s^{\top}\right]$ and $\mathbb{E}_{\mathcal{Y}_{t}}[$.$] denote the conditional expecta-$ tion given $\mathcal{Y}_{t}$. Hereafter we let $\mathbb{N}_{+}:=\{1,2, \ldots\}$ and $\mathbb{N}:=$ $\mathbb{N}_{+} \cup\{0\}$. We let $\operatorname{tr}(\cdot)$ denote the trace of a square matrix, $\|\cdot\|_{p}$ denote the standard $p$-norm, and simply $\|\cdot\|$ denote the Euclidean norm. We denote by $\|s\|_{M}:=\sqrt{s^{\top} M s}$ the weighted 2-norm, for $M=M^{\top} \geqslant 0$. In a Euclidean space we denote by $\mathcal{B}_{r}$ the closed Euclidean ball of radius $r$ centered at the origin. For any two matrices $A$ and $B$ of compatible dimensions, we denote by $\mathfrak{R}_{k}(A, B)$ the $k$-th step reachability matrix $\mathfrak{R}_{k}(A, B):=\left[\begin{array}{llll}A^{k-1} B & \cdots & A B & B\end{array}\right]$. For any matrix $M$, we let $\sigma_{\min }$ and $\sigma_{\max }$ be its minimal and maximal singular values, respectively. We let $(M)_{i_{1}: i_{2}}$ denote the sub-matrix obtained by selecting the rows $i_{1}$ through $i_{2}$ of $M$ and $(M)_{i}$ denote the $i$-th row of $M$. For any positive real number $s,\lceil s\rceil$ denotes the smallest integer that upper-bounds $s$.

\section{Problem Setup}

Consider the following affine discrete-time stochastic dynamical model:

$$
\begin{aligned}
x_{t+1} & =A x_{t}+B u_{t}+w_{t}, \\
y_{t} & =C x_{t}+v_{t}
\end{aligned}
$$

where $t \in \mathbb{N}, x_{t} \in \mathbb{R}^{n}$ is the state, $u_{t} \in \mathbb{R}^{m}$ is the control input, $y_{t} \in \mathbb{R}^{p}$ is the output, $w_{t} \in \mathbb{R}^{n}$ is a random process noise, $v_{t} \in \mathbb{R}^{p}$ is a random measurement noise, and $A$, $B$, and $C$ are known matrices. We posit the following standing assumption:

\section{Assumption 1}

(i) The pair $(A, B)$ is stabilizable [6, Chapter 12].

(ii) The matrix $A$ is Lyapunov stable [6, Chapter 12], i.e., the eigenvalues $\left\{\lambda_{i}(A) \mid i=1, \ldots, n\right\}$ lie in the closed unit disc, and those eigenvalues $\lambda_{j}(A)$ with $\left|\lambda_{j}(A)\right|=1$ have equal algebraic and geometric multiplicities.

(iii) The initial condition and the process and measurement noise vectors are mutually independent and normally distributed, i.e., $x_{0} \sim \mathcal{N}\left(0, \Sigma_{x_{0}}\right), w_{t} \sim$ $\mathcal{N}\left(0, \Sigma_{w}\right)$, and $v_{t} \sim \mathcal{N}\left(0, \Sigma_{v}\right)$, with $\Sigma_{w}>0$ and $\Sigma_{v}>0$.

(iv) $\left(A, \Sigma_{w}^{1 / 2}\right)$ is controllable and $(A, C)$ is observable.

(v) The control inputs satisfy

$$
\left\|u_{t}\right\|_{\infty} \leqslant U_{\max } \quad \forall t \in \mathbb{N} .
$$

Without loss of generality, we assume that $A$ is given in real Jordan canonical form. Indeed, given a linear system described by the system matrices $(\tilde{A}, \tilde{B})$, there exists a coordinate transformation in the state-space that brings the pair $(\tilde{A}, \tilde{B})$ to the pair $(A, B)$, where $A$ is in real Jordan form [23, p. 150]. In particular, choosing a suitable ordering of the Jordan blocks, we can ensure that the pair $(A, B)$ has the form $\left(\left[\begin{array}{cc}A_{s} & 0 \\ 0 & A_{o}\end{array}\right],\left[\begin{array}{l}B_{s} \\ B_{o}\end{array}\right]\right)$, where $A_{s} \in \mathbb{R}^{n_{s} \times n_{s}}$ is Schur stable, and $A_{o} \in \mathbb{R}^{n_{o} \times n_{o}}$ has its eigenvalues on the unit circle. By Assumption 1-(ii), $A_{o}$ is therefore block-diagonal with the diagonal blocks being either \pm 1 , or $2 \times 2$ rotation matrices. As a consequence, $A_{o}$ is orthogonal. Moreover, since $(A, B)$ is stabilizable, the pair $\left(A_{o}, B_{o}\right)$ must be reachable in a number of steps $\kappa \leqslant n_{o}$ that depends on the dimension of $A_{o}$. Therefore, we can start by considering that the state equation (1a) has the form

$$
\left[\begin{array}{l}
x_{t+1}^{s} \\
x_{t+1}^{o}
\end{array}\right]=\left[\begin{array}{l}
A_{s} x_{t}^{s} \\
A_{o} x_{t}^{o}
\end{array}\right]+\left[\begin{array}{l}
B_{s} \\
B_{o}
\end{array}\right] u_{t}+\left[\begin{array}{l}
w_{t}^{s} \\
w_{t}^{o}
\end{array}\right]
$$

where $A_{s}$ is Schur stable, $A_{o}$ is orthogonal, and there exists a nonnegative integer $\kappa \leqslant n_{o}$ such that the subsystem $\left(A_{o}, B_{o}\right)$ is reachable in $\kappa$ steps. This reachability index $\kappa$ is fixed throughout the rest of the article.

For each $t \in \mathbb{N}$, let $\mathcal{Y}_{t}:=\left\{y_{0}, \cdots, y_{t}\right\}$ denote the set of output observations up to time $t$. Fix a prediction horizon $N \in \mathbb{N}_{+}$, with $N \geqslant \kappa$, and define the cost $\mathcal{J}_{t}$ as

$$
\mathcal{J}_{t}=\mathbb{E}_{\mathcal{Y}_{t}}\left[\sum_{k=0}^{N-1}\left(\left\|x_{t+k}\right\|_{Q_{k}}^{2}+\left\|u_{t+k}\right\|_{R_{k}}^{2}\right)+\left\|x_{t+N}\right\|_{Q_{N}}^{2}\right]
$$

where $Q_{k}=Q_{k}^{\top} \geqslant 0, Q_{N}=Q_{N}^{\top} \geqslant 0$, and $R_{k}=R_{k}^{\top} \geqslant 0$ are given matrices of appropriate dimension, for $k=$ $0, \ldots, N-1$.

The evolution of the system (1a)-(1b) over a single prediction horizon $N$, starting at $t$, can be described in a compact form as

$$
X_{t}=\mathcal{A} x_{t}+\mathcal{B} U_{t}+\mathcal{D} W_{t}, \quad Y_{t}=\mathcal{C} X_{t}+V_{t},
$$

where $X_{t}=\left[\begin{array}{c}x_{t} \\ x_{t+1} \\ \vdots \\ x_{t+N}\end{array}\right], U_{t}=\left[\begin{array}{c}u_{t} \\ u_{t+1} \\ \vdots \\ u_{t+N-1}\end{array}\right], W_{t}=$

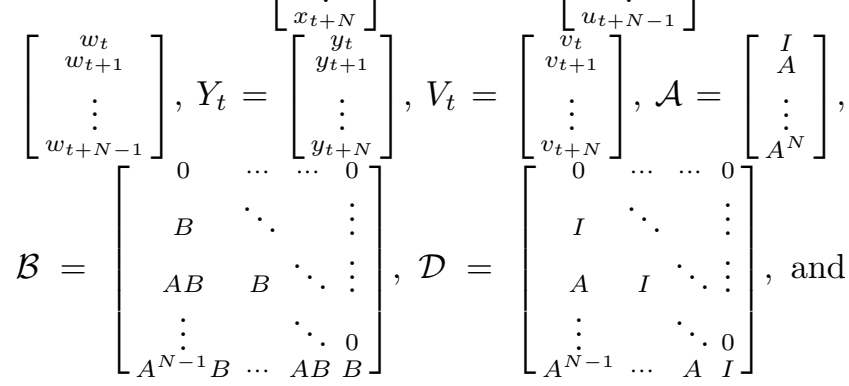


$\mathcal{C}=\operatorname{diag}\{C, \cdots, C\}$. The cost function (4) at time $t$ can also be written compactly as

$$
\mathcal{J}_{t}=\mathbb{E}_{\mathcal{Y}_{t}}\left[\left\|X_{t}\right\|_{\mathcal{Q}}^{2}+\left\|U_{t}\right\|_{\mathcal{R}}^{2}\right]
$$

where $\mathcal{Q}=\operatorname{diag}\left\{Q_{0}, \cdots, Q_{N}\right\}$ and $\mathcal{R}=\operatorname{diag}\left\{R_{0}\right.$, $\left.\cdots, R_{N-1}\right\}$. The cost $\mathcal{J}_{t}$ in (4) is a conditional expectation given the observations up to time $t$, the evaluation of which requires the conditional density $f\left(x_{t} \mid \mathcal{Y}_{t}\right)$ of the state given the previous and current measurements. For $t, s \in \mathbb{N}$, define $\hat{x}_{t \mid s}=\mathbb{E}_{\mathcal{Y}_{s}}\left[x_{t}\right]$ and $P_{t \mid s}=\mathbb{E}_{\mathcal{Y}_{s}}\left[\left(x_{t}-\hat{x}_{t \mid s}\right)\left(x_{t}-\hat{x}_{t \mid s}\right)^{\top}\right]$.

The following result is a slight extension of the standard Kalman filter. A proof may be found in [28, p.102].

Proposition 2 Let Assumption 1-(iii) hold and assume that $u_{t}$ is a deterministic function of $\mathcal{Y}_{t}$. Then $f\left(x_{t} \mid \mathcal{Y}_{t}\right)$ and $f\left(x_{t+1} \mid \mathcal{Y}_{t}\right)$ are the probability densities of Gaussian distributions $\mathcal{N}\left(\hat{x}_{t \mid t}, P_{t \mid t}\right)$ and $\mathcal{N}\left(\hat{x}_{t+1 \mid t}, P_{t+1 \mid t}\right)$, respectively, with $P_{t \mid t} \geqslant 0$ and $P_{t+1 \mid t} \geqslant 0$. For $t=-1,0,1,2, \ldots$, their conditional means and covariances can be computed iteratively starting at $\left(\hat{x}_{0 \mid-1}, P_{0 \mid-1}\right):=\left(0, \Sigma_{x_{0}}\right)$, as follows:

$$
\begin{aligned}
\hat{x}_{t+1 \mid t+1}= & \hat{x}_{t+1 \mid t} \\
+P_{t+1 \mid t} & C^{\top}\left(C P_{t+1 \mid t} C^{\top}+\Sigma_{v}\right)^{-1}\left(y_{t+1}-C \hat{x}_{t+1 \mid t}\right) \\
P_{t+1 \mid t+1}= & P_{t+1 \mid t} \\
& -P_{t+1 \mid t} C^{\top}\left(C P_{t+1 \mid t} C^{\top}+\Sigma_{v}\right)^{-1} C P_{t+1 \mid t} \\
\hat{x}_{t+1 \mid t}= & A \hat{x}_{t \mid t}+B u_{t} \\
P_{t+1 \mid t}= & A P_{t \mid t} A^{\top}+\Sigma_{w}
\end{aligned}
$$

Proposition 2 states that the conditional mean and covariances of $x_{t}$ can be propagated by an iterative algorithm which resembles the Kalman filter. In particular, the matrix $P_{t \mid t}$ together with $\hat{x}_{t \mid t}$ characterize the conditional density $f\left(x_{t} \mid \mathcal{Y}_{t}\right)$, which is needed in the computation of the cost (4) (or equivalently (6)). We note here that in the receding horizon control case considered in this paper $u_{t}$ will be a nonlinear function of $\left\{y_{0}, \cdots, y_{t}\right\}$; therefore we cannot assume that all the probability distributions in the problem are Gaussian as in the case of LQG; in fact, the a priori distributions of $x_{t}$ and of $\mathcal{Y}_{t}$ are not. Hereafter, we shall denote for notational convenience by $\hat{x}_{t}$ the estimate $\hat{x}_{t \mid t}$, and let

$$
\hat{x}_{t}=\left[\left(\hat{x}_{t}^{s}\right)^{\top}\left(\hat{x}_{t}^{o}\right)^{\top}\right]^{\top},
$$

which corresponds to the Jordan decomposition in (3). Let $K_{t}:=\left(A P_{t \mid t} A^{\top}+\Sigma_{w}\right) C^{\top}\left(C\left(A P_{t \mid t} A^{\top}+\Sigma_{w}\right) C^{\top}+\right.$ $\left.\Sigma_{v}\right)^{-1}$ and define $\Gamma_{t}:=I-K_{t} C$ and $\Phi_{t}:=\Gamma_{t} A$. Then, we can write the estimation error vector over a single prediction horizon $N$ as

$$
E_{t}:=X_{t}-\hat{X}_{t}=\mathcal{F}_{t} e_{t}+\mathcal{G}_{t} W_{t}-\mathcal{H}_{t} V_{t},
$$

where $e_{t}=x_{t}-\hat{x}_{t}, \quad \hat{X}_{t}=\left[\begin{array}{c}\hat{x}_{t} \\ \vdots \\ \hat{x}_{t+N}\end{array}\right], \mathcal{F}_{t}=\left[\begin{array}{c}I \\ \Phi_{t} \\ \Phi_{t+1} \cdot \Phi_{t} \\ \vdots \\ \Phi_{t+N-1} \cdots \Phi_{t}\end{array}\right]$

$$
\begin{aligned}
& \mathcal{G}_{t}=\left[\begin{array}{llll}
0 & \cdots & 0 & 0 \\
\Gamma_{t} & \cdots & 0 & 0 \\
\Phi_{t+1} \Gamma_{t} & \cdots & 0 & 0 \\
\vdots & & \vdots & \vdots \\
\Phi_{t+N-2} \cdots \Phi_{t+1} \Gamma_{t} & \cdots & \Gamma_{t+N-2} & 0 \\
\Phi_{t+N-1} \cdots \Phi_{t+1} \Gamma_{t} & \cdots & \Phi_{t+N-1} \Gamma_{t+N-2} & \Gamma_{t+N-1}
\end{array}\right] \\
& \mathcal{H}_{t}=\left[\begin{array}{lllll}
0 & 0 & \cdots & 0 & 0 \\
0 & K_{t} & \cdots & 0 & 0 \\
0 & \Phi_{t+1} K_{t} & \cdots & 0 & 0 \\
\vdots & \vdots & & \vdots & \vdots \\
0 & \Phi_{t+N-2} \cdots \Phi_{t+1} K_{t} & \cdots & K_{t+N-2} & 0 \\
0 & \Phi_{t+N-1} \cdots \Phi_{t+1} K_{t} & \cdots & \Phi_{t+N-1} K_{t+N-2} & K_{t+N-1}
\end{array}\right] .
\end{aligned}
$$

The innovations sequence can be written as

$$
Y_{t}-\hat{Y}_{t}=\mathcal{C} \mathcal{F}_{t} e_{t}+\mathcal{C G}_{t} W_{t}+\left(I-\mathcal{C} \mathcal{H}_{t}\right) V_{t},
$$

where $\hat{Y}_{t}:=\mathcal{C} \hat{X}_{t}$. Consequently, the innovations sequence over the prediction horizon is independent of the input vector $U_{t}$. Also, under the forgoing assumptions, the error vector $e_{t}$ is a Gaussian random variable with mean zero and variance $P_{t \mid t}$.

\subsection{Optimization Problem and Control Policies}

We would like to minimize the cost (4) over the class of all causal feedback policies. However, this optimization problem is extremely difficult to solve in general $[7,8]$. Therefore, we restrict attention to a subclass of causal feedback policies for which the optimization problem is tractable. Guided by our earlier approach in $[20,16,22,21]$ and given a control horizon $N_{c} \geqslant 1$ and a prediction horizon $N \geqslant N_{c}$, we would like to periodically minimize the cost (4) at times $t=0, N_{c}, 2 N_{c}, \cdots$ over the following class of control policies

$$
u_{t+\ell}=\eta_{t+\ell}+\sum_{i=0}^{\ell} \theta_{t+\ell, t+i} \varphi_{i}\left(y_{t+i}-\hat{y}_{t+i}\right),
$$

where $\ell=0,1, \cdots, N-1, \hat{y}_{i}=C \hat{x}_{i}$ is the output of the estimator, and for any vector $z=\left(z_{1}, \cdots, z_{p}\right) \in \mathbb{R}^{p}$, $\varphi_{i}(z)=\left(\varphi_{i, 1}\left(z_{1}\right), \cdots, \varphi_{i, p}\left(z_{p}\right)\right)$, where $\varphi_{i, j}: \mathbb{R} \rightarrow \mathbb{R}$ is any function with $\sup _{s \in \mathbb{R}}\left|\varphi_{i, j}(s)\right| \leqslant \varphi_{\max }<\infty$ for some $\varphi_{\max }>0$. The feedback gains $\theta_{\ell, i} \in \mathbb{R}^{m \times p}$ and the affine terms $\eta_{\ell} \in \mathbb{R}^{m}$ are the decision variables. The value of $u_{t+\ell}$ in (14) depends on the values of the measured outputs from the beginning of the prediction horizon at time $t$ up to time $t+\ell$ only, which requires finite memory. Note that we have chosen to saturate the measurements we obtain from the vectors $\left(y_{i}-\hat{y}_{i}\right)$ before employing 
them in the control policy. This allows us to consider unbounded noise and yet ensure bounded policies; neither the process noise nor the measurement noise distributions are defined over a compact domain, in contrast to robust deterministic receding horizon control [35] or other stochastic receding horizon control approaches as in [13]. Moreover, the choice of element-wise saturation functions $\varphi_{i}(\cdot)$ is left open. As such, we can accommodate standard saturation, piecewise linear, and sigmoidal functions, to name a few. The control policy (14) at time $t$ can be compactly written as

$$
U_{t}=\boldsymbol{\eta}_{t}+\boldsymbol{\Theta}_{t} \varphi\left(Y_{t}-\hat{Y}_{t}\right)
$$

where $\boldsymbol{\Theta}_{t}$ has the following (causal) lower block triangular structure

$$
\boldsymbol{\Theta}_{t}:=\left[\begin{array}{cccc}
\theta_{t, t} & 0 & \cdots & 0 \\
\theta_{t+1, t} & \theta_{t+1, t+1} & & \vdots \\
\vdots & \vdots & \ddots & 0 \\
\theta_{t+N-1, t} & \theta_{t+N-1, t+1} & \ldots & \theta_{t+N-1, t+N-1}
\end{array}\right]
$$

$\boldsymbol{\eta}_{t}:=\left[\begin{array}{c}\eta_{t} \\ \eta_{t+1} \\ \vdots \\ \eta_{t+N-1}\end{array}\right]$, and $\varphi\left(Y_{t}-\hat{Y}_{t}\right):=\left[\begin{array}{c}\varphi_{0}\left(y_{t}-\hat{y}_{t}\right) \\ \vdots \\ \varphi_{N-1}\left(y_{t+N-1}-\hat{y}_{t+N-1}\right)\end{array}\right]$ Since the innovations vector $Y_{t}-\hat{Y}_{t}$ in (13) is not a function of $\boldsymbol{\eta}_{t}$ and $\boldsymbol{\Theta}_{t}$, the control inputs $U_{t}$ in (14) remain affine in the decision variables. This fact is important to show convexity of the optimization problem, as will be seen in the next section. Finally, the constraint (2) can be rewritten as:

$$
\left\|U_{t}\right\|_{\infty} \leqslant U_{\max } \quad \forall t=0, N_{c}, 2 N_{c}, \cdots
$$

Summarizing, the optimization problem to be solved periodically at times $t=0, N_{c}, 2 N_{c}, \cdots$ is given by

$$
\min _{\left(\boldsymbol{\eta}_{t}, \Theta_{t}\right)}\left\{\mathcal{J}_{t} \mid(5),(15),(16),(17)\right\}
$$

\section{Main Results}

Even if problem (18) is successively feasible every $N_{c}$ steps, in general the resulting control actions do not guarantee stability of the resulting receding horizon controller. Unlike standard deterministic stability arguments utilized in MPC, see, for example, [35], we cannot assume the existence of a compact robust positively invariant terminal region, since the process noise sequence does not have a compact support. Instead, we introduce an additional stability constraint which, if recursively feasible, renders the state of the closed-loop system mean-square bounded. Guided by the argument in [39], we then show that this constraint is indeed recursively feasible.

For $t=0, N_{c}, 2 N_{c}, \cdots$, the state estimate at time $t+N_{c}$ can be written as

$$
\hat{x}_{t+N_{c}}=A^{N_{c}} \hat{x}_{t}+\Re_{N_{c}}(A, B)\left[\begin{array}{c}
u_{t} \\
\vdots \\
u_{t+N_{c}-1}
\end{array}\right]+\Xi_{t},
$$

where $\mathfrak{R}_{N_{c}}(A, B)$ is the reachability matrix as defined earlier and $\Xi_{t}$ is defined as

$$
\begin{aligned}
\Xi_{t}:= & {\left[\begin{array}{llll}
A^{N_{c}-1} K_{t} C A & A^{N_{c}-2} K_{t+1} C A & \cdots & K_{t+N_{c}-1} C A
\end{array}\right]\left[\begin{array}{c}
e_{t} \\
\vdots \\
e_{t+N_{c}-1}
\end{array}\right] } \\
& +\left[\begin{array}{llll}
A^{N_{c}-1} K_{t} C & A^{N_{c}-2} K_{t+1} C & \cdots & K_{t+N_{c}-1} C
\end{array}\right]\left[\begin{array}{c}
w_{t} \\
\vdots \\
w_{t+N_{c}-1}
\end{array}\right] \\
& +\left[\begin{array}{llll}
A^{N_{c}-1} K_{t} & A^{N_{c}-2} K_{t+1} & \cdots & K_{t+N_{c}-1}
\end{array}\right]\left[\begin{array}{c}
v_{t+1} \\
\vdots \\
v_{t+N_{c}}
\end{array}\right] \cdot(20)
\end{aligned}
$$

In order to show boundedness of the state variance, we require that the $N_{c}$-step iteration (19) has bounded variance. However, this estimate has the term $\Xi_{t}$, which involves the error in the state estimation process as well as the process and measurement noise vectors. This term $\Xi_{t}$ may be viewed as 'noise' entering the system, with bounded fourth moment. In particular, we require that there exists, at least after some time $T^{\prime}$, a uniform bound on its first moment. This is captured by the following Proposition.

Proposition 3 There exists an integer $T^{\prime}$ and a positive constant $\zeta$, depending on the given problem parameters, such that

$$
\mathbb{E}_{\mathcal{Y}_{t}}\left[\left\|\Xi_{t}\right\|\right] \leqslant \zeta \quad \text { for all } t \geqslant T^{\prime}
$$

Using the constant $\zeta$, we now require the following "drift condition" to be satisfied: for any chosen constant $\varepsilon>0$ and for every $t=0, N_{c}, 2 N_{c}, \cdots, U_{t} \in \mathbb{U}$ is designed such that the following condition is satisfied

$$
\begin{gathered}
\left\|A_{o}^{N_{c}} \hat{x}_{t}^{o}+\Re_{N_{c}}\left(A_{o}, B_{o}\right)\left[\begin{array}{c}
u_{t} \\
\vdots \\
u_{t+N_{c}-1}
\end{array}\right]\right\| \leqslant\left\|\hat{x}_{t}^{o}\right\|-\left(\zeta+\frac{\varepsilon}{2}\right) \\
\text { whenever }\left\|\hat{x}_{t}^{o}\right\| \geqslant \zeta+\varepsilon .
\end{gathered}
$$

As will be shown later, condition (22) above guarantees that on average the state norm contracts every $N_{c}$ steps, a crucial ingredient towards showing meansquare boundedness of the closed-loop system. Moreover, $N_{c}$ needs to be chosen appropriately (depending on the reachability index $\kappa$ ) in order to ensure that the constraint is feasible. Note that $\left[\begin{array}{c}u_{t} \\ \vdots \\ u_{t+N_{c}-1}\end{array}\right]=\left(\boldsymbol{\eta}_{t}\right)_{1: N_{c} m}+$ $\left(\boldsymbol{\Theta}_{t}\right)_{1: N_{c} m} \varphi(Y-\hat{Y})$. (For notational convenience, we have retained $\varphi(Y-\hat{Y})$ with the knowledge that the matrix $\left(\boldsymbol{\Theta}_{t}\right)_{1: N_{c} m}$ causally selects the first $N_{c}$ output vectors as they become available, see (16).) We augment 
problem (18) with the stability constraint (22) to obtain

$$
\min _{\left(\boldsymbol{\eta}_{t}, \Theta_{t}\right)}\left\{\mathcal{J}_{t} \mid(5),(15),(16),(17),(22)\right\} \text {. }
$$

The ingredients of our stochastic receding horizon control problem corresponding to (23) are summarized in Algorithm 1.

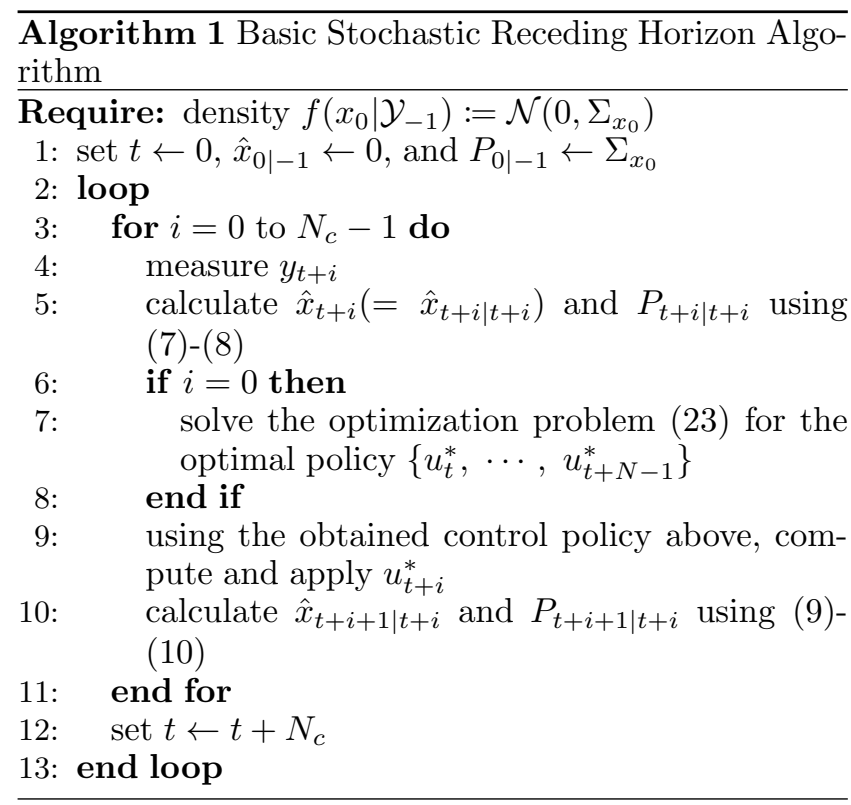

\section{Assumption 4 We require that:}

(i) The control and prediction horizons satisfy $N \geqslant$ $N_{c}=\kappa$, where $\kappa$ is the reachability index of the orthogonal subsystem $\left(A_{o}, B_{o}\right)$ in (3).

(ii) The control authority $U_{\max } \geqslant U_{\max }^{*}$, where $U_{\max }^{*}$ : $=\sigma_{\min }\left(\mathfrak{R}_{N_{c}}\left(A_{o}, B_{o}\right)\right)^{-1}\left(\zeta+\frac{\varepsilon}{2}\right)$ and $\mathfrak{R}_{N_{c}}\left(A_{o}, B_{o}\right)$ is the $N_{c}$-step reachability matrix of the orthogonal subsystem.

In fact, choosing any control horizon $N_{c} \geqslant \kappa$ turns out to be sufficient in order to have a feasible control vector $U_{t}$ for problem (23) with an upper bound $U_{\max }=U_{\max }^{*}$; however, we will take $N_{c}=\kappa$ for simplicity.

Theorem 5 Consider the system (1a)-(1b), and suppose that Assumptions 1 and 4 hold. Then:

(i) For every time $t=0, N_{c}, 2 N_{c}, \cdots$, the optimization problem (23) in Algorithm 1 is convex and can be conservatively approximated and solved via the following globally (hence recursively) feasible secondorder cone program (SOCP):

$$
\begin{aligned}
& \underset{\left(z_{1}, z_{2}, z_{3}, \boldsymbol{\eta}_{t}, \boldsymbol{\Theta}_{t}\right)}{\operatorname{minimize}} z_{1} \\
& \text { subject to } \\
& \left\|\boldsymbol{\eta}_{t}+\boldsymbol{\Theta}_{t} \Lambda_{t}^{\varphi}\right\|_{\mathcal{M}}^{2}+\operatorname{tr}\left(\boldsymbol{\Theta}_{t}^{\top} \mathcal{M} \boldsymbol{\Theta}_{t}\left(\Lambda_{t}^{\varphi \varphi}-\Lambda_{t}^{\varphi} \Lambda_{t}^{\varphi \top}\right)\right)+ \\
& 2 \hat{x}_{t}^{\top} \mathcal{A}^{\top} \mathcal{Q} \mathcal{B} \boldsymbol{\eta}_{t}+2 \operatorname{tr}\left(\boldsymbol{\Theta}_{t}^{\top} \mathcal{B}^{\top} \mathcal{Q}\left(\mathcal{D} \Lambda_{t}^{w \varphi}+\mathcal{A} \Lambda_{t}^{x \varphi}\right)\right) \leqslant z_{1}
\end{aligned}
$$

$$
\left|\left(\boldsymbol{\eta}_{t}\right)_{i}\right|+\left\|\left(\boldsymbol{\Theta}_{t}\right)_{i}\right\|_{1} \varphi_{\max } \leqslant U_{\max } \quad \forall i=1, \cdots, N m,
$$

$$
\left.\begin{array}{l}
\left\|A_{o}^{N_{c}} \hat{x}_{t}^{o}+\Re_{N_{c}}\left(A_{o}, B_{o}\right)\left(\boldsymbol{\eta}_{t}\right)_{1: N_{c} m}\right\| \leqslant z_{2} \\
\left\|\Re_{N_{c}}\left(A_{o}, B_{o}\right)\left(\boldsymbol{\Theta}_{t}\right)_{1: N_{c} m}\right\|_{\infty} \leqslant z_{3} \\
z_{2}+\sqrt{n_{o}} \varphi_{\max } z_{3} \leqslant\left\|\hat{x}_{t}^{o}\right\|-\left(\zeta+\frac{\varepsilon}{2}\right)
\end{array}\right\}\left\|\hat{x}_{t}^{o}\right\| \geqslant \zeta+\varepsilon
$$

the structure of $\boldsymbol{\Theta}_{t}$ in $(16)$,

where $\mathcal{M}:=\mathcal{R}+\mathcal{B}^{\top} \mathcal{Q B}$, and

$$
\begin{aligned}
\Lambda_{t}^{\varphi} & :=\mathbb{E}_{\mathcal{Y}_{t}}\left[\varphi\left(Y_{t}-\hat{Y}_{t}\right)\right], \Lambda_{t}^{x \varphi}:=\mathbb{E}_{\mathcal{Y}_{t}}\left[x_{t} \varphi\left(Y_{t}-\hat{Y}_{t}\right)^{\top}\right] \\
\Lambda_{t}^{w \varphi} & :=\mathbb{E}_{\mathcal{Y}_{t}}\left[W \varphi\left(Y_{t}-\hat{Y}_{t}\right)^{\top}\right] \\
\Lambda_{t}^{\varphi \varphi} & :=\mathbb{E}_{\mathcal{Y}_{t}}\left[\varphi\left(Y_{t}-\hat{Y}_{t}\right) \varphi\left(Y_{t}-\hat{Y}_{t}\right)^{\top}\right] .
\end{aligned}
$$

(ii) The application of Algorithm 1 via the SOCP approximation in part (i) above renders the closed-loop system mean-square bounded, i.e., for any initial $\mathcal{Y}_{0}$, there exists a (computable) finite constant $\gamma>0$, depending on the given problem parameters, such that

$$
\sup _{t \in \mathbb{N}} \mathbb{E}_{\mathcal{Y}_{0}}\left[\left\|x_{t}\right\|^{2}\right] \leqslant \gamma \text {. }
$$

In practice, it may be also of interest to further impose constraints both on the state and the input vectors. For example, one may be interested in imposing linear and / or quadratic constraints on the state of the form

$$
\mathbb{E}_{\mathcal{Y}_{t}}\left[\left\|X_{t}\right\|_{\mathcal{S}}^{2}+\mathcal{L}^{\top} X_{t}\right] \leqslant \alpha_{t}
$$

where $\mathcal{S}=\mathcal{S}^{\top} \geqslant 0$ and $\alpha_{t}>0$. Moreover, expected energy expenditure constraints can be posed as follows

$$
\mathbb{E}_{\mathcal{Y}_{t}}\left[\left\|U_{t}\right\|_{\tilde{\mathcal{S}}}^{2}\right] \leqslant \beta_{t}
$$

where $\tilde{\mathcal{S}}=\tilde{\mathcal{S}}^{\top} \geqslant 0$ and $\beta_{t}>0$. In the absence of hard input constraints, such expectation-type constraints are commonly used in the stochastic MPC $[38,17]$ and in stochastic optimization in the form of integrated chance constraints $[26,27]$. This is partly because it is not possible, without posing further restrictions on the boundedness of the process noise $w_{t}$, to ensure that hard constraints on the state are satisfied. For example, in the standard LQG setting nontrivial hard constraints on the system state would generally be violated with nonzero probability. Moreover, in contrast to chance constraints where a bound is imposed on the probability of constraint violation, expectation-type constraints tend to give rise to convex optimization problems under weak assumptions $[17,26,27]$. We can augment problem (23) with the constraints (30) and (31) to obtain

$$
\min _{\left(\boldsymbol{\eta}_{t}, \Theta_{t}\right)}\left\{\mathcal{J}_{t} \mid(5),(15),(16),(17),(22),(30),(31)\right\} .
$$


Notice that the constraints (30) and (31) are not necessarily feasible at time $t$ for any choice of parameters $\alpha_{t}$ and $\beta_{t}$. As such, problem (32) may become infeasible over time if we simply apply Algorithm 1 . We therefore modify step 7 in Algorithm 1 to generate Algorithm 2. In this new version of the algorithm, problem (32) is either feasible at step 7 with the given $\alpha_{t}$ and $\beta_{t}$, or a bisection search is implemented (steps 12 to 26), with $\alpha_{t}$ and $\beta_{t}$ as lower bounds, and upper bounds

$$
\begin{aligned}
\alpha_{t}^{*} & :=3 \operatorname{tr}\left(\mathcal{A}^{\top} \mathcal{S} \mathcal{A} \mathbb{E}_{\mathcal{Y}_{t}}\left[x_{t} x_{t}^{\top}\right]+\mathcal{D}^{\top} \mathcal{S} \mathcal{D} \Sigma_{w}\right)+\mathcal{L}^{\top} \mathcal{A} \hat{x}_{t} \\
& +3 N m \sigma_{\max }\left(\mathcal{B}^{\top} \mathcal{S B}\right) U_{\max }^{2}+\left\|\mathcal{L}^{\top} \mathcal{B}\right\|_{1} U_{\max } \\
\beta_{t}^{*} & :=N m \sigma_{\max }(\tilde{\mathcal{S}}) U_{\max }^{2}
\end{aligned}
$$

that guarantee feasibility. The search is iterated until the change in $\alpha$ and $\beta$ falls below a prespecified precision number $\delta$ or a maximal number of iterations $\bar{\nu}$ is reached, which is used to keep the computational burden limited.

Corollary 6 Consider the system (1a)-(1b), and suppose that Assumptions 1 and 4 hold. Then:

(i) For every time $t=0, N_{c}, 2 N_{c}, \cdots$ the optimization problem (32) in Algorithm 2 is convex and can be conservatively approximated and solved via the following globally (hence recursively) feasible (at either step 7 or step 13) second-order cone program (SOCP):

$$
\begin{aligned}
& \underset{\left(z_{1}, z_{2}, z_{3}, \boldsymbol{\eta}_{t}, \boldsymbol{\Theta}_{t}\right)}{\operatorname{minimize}} \quad z_{1} \\
& \text { subject to } \\
& \left\|\boldsymbol{\eta}_{t}+\boldsymbol{\Theta}_{t} \Lambda_{t}^{\varphi}\right\|_{\mathcal{M}}^{2}+\operatorname{tr}\left(\boldsymbol{\Theta}_{t}^{\top} \mathcal{M} \Theta_{t}\left(\Lambda_{t}^{\varphi \varphi}-\Lambda_{t}^{\varphi} \Lambda_{t}^{\varphi \top}\right)\right)+ \\
& 2 \hat{x}_{t}^{\top} \mathcal{A}^{\top} \mathcal{Q} \mathcal{B} \boldsymbol{\eta}_{t}+2 \operatorname{tr}\left(\boldsymbol{\Theta}_{t}^{\top} \mathcal{B}^{\top} \mathcal{Q}\left(\mathcal{D} \Lambda_{t}^{w \varphi}+\mathcal{A} \Lambda_{t}^{x \varphi}\right)\right) \leqslant z_{1} \\
& \left|\left(\boldsymbol{\eta}_{t}\right)_{i}\right|+\left\|\left(\boldsymbol{\Theta}_{t}\right)_{i}\right\|_{1} \varphi_{\max } \leqslant U_{\max } \quad \forall i=1, \cdots, N m, \\
& \left\|A_{o}^{N_{c}} \hat{x}_{t}^{o}+\Re_{N_{c}}\left(A_{o}, B_{o}\right)\left(\boldsymbol{\eta}_{t}\right)_{1: N_{c} m}\right\| \leqslant z_{2}{ }_{\text {whenever }} \\
& \left\|\Re_{N_{c}}\left(A_{o}, B_{o}\right)\left(\boldsymbol{\Theta}_{t}\right)_{1: N_{c} m}\right\|_{\infty} \leqslant z_{3} \\
& z_{2}+\sqrt{n_{o}} \varphi_{\max } z_{3} \leqslant\left\|\hat{x}_{t}^{o}\right\|-\left(\zeta+\frac{\varepsilon}{2}\right) \\
& \int\left\|\hat{x}_{t}^{o}\right\| \geqslant \zeta+\varepsilon \\
& \left\|\boldsymbol{\eta}_{t}+\boldsymbol{\Theta}_{t} \Lambda_{t}^{\varphi}\right\|_{\mathcal{B}^{\top} \mathcal{S B}}^{2}+\operatorname{tr}\left(\boldsymbol{\Theta}_{t}^{\top} \mathcal{B}^{\top} \mathcal{S B} \boldsymbol{\Theta}_{t}\left(\Lambda_{t}^{\varphi \varphi}-\Lambda_{t}^{\varphi} \Lambda_{t}^{\varphi \mathrm{\top}}\right)\right) \\
& +2 \hat{x}_{t}^{\top} \mathcal{A}^{\top} \mathcal{S} \mathcal{B} \boldsymbol{\eta}_{t}+2 \operatorname{tr}\left(\Theta_{t}^{\top} \mathcal{B}^{\top} \mathcal{S}\left(\mathcal{D} \Lambda_{t}^{w \varphi}+\mathcal{A} \Lambda_{t}^{x \varphi}\right)\right) \\
& +\mathcal{L}^{\top} \mathcal{B}\left(\boldsymbol{\eta}_{t}+\boldsymbol{\Theta}_{t} \Lambda_{t}^{\varphi}\right)+\operatorname{tr}\left(\mathcal{A}^{\top} \mathcal{S} \mathcal{A} \mathbb{E}_{\mathcal{Y}_{t}}\left[x_{t} x_{t}^{\top}\right]\right) \\
& +\operatorname{tr}\left(\mathcal{D}^{\top} \mathcal{S D} \Sigma_{w}\right)+\mathcal{L}^{\top} \mathcal{A} \hat{x}_{t} \leqslant \alpha_{t} \\
& \left\|\boldsymbol{\eta}_{t}+\boldsymbol{\Theta}_{t} \Lambda_{t}^{\varphi}\right\|_{\tilde{\mathcal{S}}}^{2}+\operatorname{tr}\left(\boldsymbol{\Theta}_{t}^{\top} \tilde{\mathcal{S}} \boldsymbol{\Theta}_{t}\left(\Lambda_{t}^{\varphi \varphi}-\Lambda_{t}^{\varphi} \Lambda_{t}^{\varphi \mathrm{\top}}\right)\right) \leqslant \beta_{t}
\end{aligned}
$$

the structure of $\boldsymbol{\Theta}_{t}$ in (16),

where all the required constant matrices are defined as in Theorem 5.

(ii) The application of Algorithm 2 via the $S O C P$ approximation in part (i) above renders the closed-loop system mean-square bounded, i.e., for any initial $\mathcal{Y}_{0}$, there exists a (computable) finite constant $\gamma>0$, de- pending on the given problem parameters, such that

$$
\sup _{t \in \mathbb{N}} \mathbb{E}_{\mathcal{Y}_{0}}\left[\left\|x_{t}\right\|^{2}\right] \leqslant \gamma \text {. }
$$

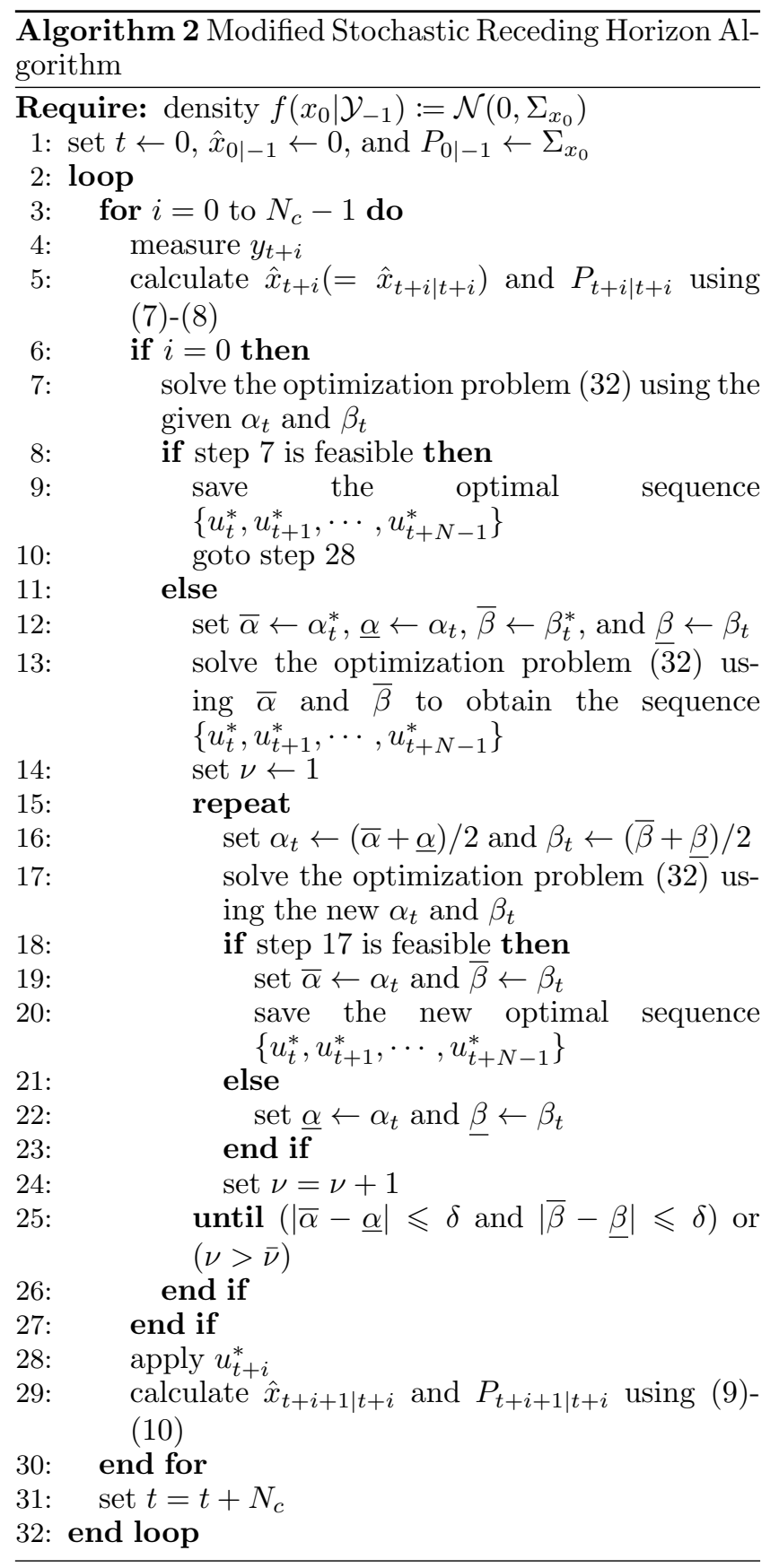

\section{Discussion}

\subsection{Recursive Feasibility}

The SOCPs solved in Theorem 5 and Corollary 6 are globally feasible, independently of the initial conditions of the plant and the estimator. As such, there is no a priori requirement for an initially feasible and invariant 
set of initial conditions, as is the case in nominal or robust receding horizon control [35]. This guarantee of recursive feasibility is shown in the proofs of Theorem 5 and Corollary 6 by providing a feasible control law that satisfies all the constraints in the SOCPs.

\subsection{Mean-Square Boundedness}

The mean-square boundedness conditions (29) and (36) provide an on-average guarantee that the state does not grow arbitrarily large. This is a weaker notion of stability than, for example, asymptotic stability or input-tostate stability (ISS) that have been utilized in nominal and robust receding horizon control, respectively. However, in the presence of possibly unbounded process and measurement noise, it is virtually impossible to guarantee that the state converges to the origin or that it is ultimately bounded in some compact set for every initial condition and every realization of the noise processes. In this case, similarly to LQG (i.e., even with unbounded control authority), mean-square boundedness is the best that can be aimed for within our setting, given the unboundedness of the noise processes and the limited control authority. The constants $\gamma$ in (29) and (36) may be computed using the derivations in the Appendix and the formulas in the Remark in [37, pp.145].

\subsection{More General Policies}

It is not difficult to show that one can also use quadratic policies of the form

$$
U_{t}=\boldsymbol{\eta}_{t}+\boldsymbol{\Theta}_{t} \varphi\left(Y_{t}-\hat{Y}_{t}\right)+\tilde{\boldsymbol{\Theta}}_{t} \tilde{\varphi}\left(Y_{t}-\hat{Y}_{t}\right),
$$

instead of (14), where $\tilde{\boldsymbol{\Theta}}_{t}$ has the same causal structure of of $\boldsymbol{\Theta}_{t}$ and $\tilde{\varphi}\left(Y_{t}-\hat{Y}_{t}\right):=\left[\tilde{\varphi}_{0}\left(y_{t}-\hat{y}_{t}\right)^{\top} \tilde{\varphi}_{0}\left(y_{t}-\hat{y}_{t}\right) \ldots\right.$ $\left.\tilde{\varphi}_{N-1}\left(y_{t+N-1}-\hat{y}_{t+N-1}\right)^{\top} \tilde{\varphi}_{N-1}\left(y_{t+N-1}-\hat{y}_{t+N-1}\right)\right]^{\top}$, with $\sup \left|\tilde{\varphi}_{i}(s)\right| \leqslant \tilde{\varphi}_{\max }<\infty$ for some $\tilde{\varphi}_{\max }>0$. The un$s \in \mathbb{R}$

derlying optimization problems (23) and (32) with the policy (37) are still convex and both Theorem 5 and Corollary 6 still apply with minor changes.

\subsection{Off-Line Computation of the $\Lambda$ Matrices}

The optimization problems (23) and (32) solved in Theorem 5 and in Corollary (6), respectively, are secondorder cone programs (SOCP) for which efficient numerical solvers are available via software packages such as yalmip [31]. As such, the optimization may be performed online. However, at any time $t=0, N_{c}, 2 N_{c}, \cdots$, our ability to solve the optimization problems in Theorem 5 and Corollary 6, respectively, hinges upon the computation of the matrices in (28).

Recall that $Y_{t}-\hat{Y}_{t}$ is the innovations sequence that was given in (13), and that $\hat{x}_{t}$ is the optimal meansquare estimate of $x_{t}$ given the history $\mathcal{Y}_{t}$. The matrices (28) may be computed by numerical integration with respect to the independent Gaussian measures of $w_{t}, \ldots w_{t+N-1}$, of $v_{t}, \ldots v_{t+N}$, and of $\left(x_{t}-\hat{x}_{t}\right)$ given $\mathcal{Y}_{t}$. Due to the large dimensionality of the integration space, this approach may be impractical for online computations. One alternative approach relies on the observation that $\Lambda_{t}^{\varphi}, \Lambda_{t}^{w \varphi}$, and $\Lambda_{t}^{\varphi \varphi}$ depend on $x_{t}$ via the difference $x_{t}-\hat{x}_{t}$. Since $x_{t}-\hat{x}_{t}$ is conditionally zeromean given $\mathcal{Y}_{t}$, we can write the dependency of (28) on the time-varying statistics of $x_{t}$ given $\mathcal{Y}_{t}$ as follows: $\Lambda_{t}^{x \varphi}\left(\hat{x}_{t}, P_{t \mid t}\right)=\Lambda_{t}^{e \varphi}\left(P_{t \mid t}\right)+\hat{x}_{t} \Lambda_{t}^{\varphi}\left(P_{t \mid t}\right)^{\top}, \Lambda_{t}^{w \varphi}\left(P_{t \mid t}\right)$, and $\Lambda_{t}^{\varphi \varphi}\left(P_{t \mid t}\right)$, where $\Lambda_{t}^{e \varphi}:=\mathbb{E}_{\mathcal{Y}_{t}}\left[\left(x_{t}-\hat{x}_{t}\right) \varphi\left(Y_{t}-\hat{Y}_{t}\right)^{\top}\right]$. In principle one may compute off-line and store the matri$\operatorname{ces} \Lambda_{t}^{e \varphi}\left(P_{t \mid t}\right), \Lambda_{t}^{\varphi}\left(P_{t \mid t}\right), \Lambda_{t}^{w \varphi}\left(P_{t \mid t}\right)$, and $\Lambda_{t}^{\varphi \varphi}\left(P_{t \mid t}\right)$, which depend on the covariance matrices $P_{t \mid t}$ but not on $\hat{x}_{t}$, and just update online the value of $\Lambda_{t}^{x \varphi}\left(\hat{x}_{t}, P_{t \mid t}\right)$ as the estimate $\hat{x}_{t}$ becomes available. However, this poses serious requirements in terms of memory. A more appealing alternative is to exploit the convergence properties of the covariance matrix $P_{t \mid t}$. The following result can be inferred, for instance, from [25, Theorem 5.1].

Proposition 7 Under Assumption 1-(iii) and (iv) the discrete-time algebraic Riccati equation in $P \in \mathbb{R}^{n \times n}$, $P=A\left[P-P C^{\top}\left(C P C^{\top}+\Sigma_{v}\right)^{-1} C P\right] A^{\top}+\Sigma_{w}$, has a unique solution $P^{*} \geqslant 0$. The sequence $P_{t+1 \mid t}$ defined by (8) and (10) converges to $P^{*}$ as t tends to $\infty$, for any initial condition $P_{0 \mid-1} \geqslant 0$.

As a consequence, from (8) one sees that $P_{t \mid t}$ converges to $P^{\circ}=P^{*}-P^{*} C^{\top}\left(C P^{*} C^{\top}+\Sigma_{v}\right)^{-1} C P^{*}$, which is the asymptotic error covariance matrix of the estimator $\hat{x}_{t}$. Thus, neglecting the initial transient, one may just compute off-line and store the matrices $\Lambda_{t}^{e \varphi}\left(P^{\circ}\right), \Lambda_{t}^{\varphi}\left(P^{\circ}\right)$, $\Lambda_{t}^{w \varphi}\left(P^{\circ}\right)$, and $\Lambda_{t}^{\varphi \varphi}\left(P^{\circ}\right)$, and just update the matrix $\Lambda_{t}^{x \varphi}\left(\hat{x}_{t}, P^{\circ}\right)$ for new values of the estimate $\hat{x}_{t}$.

\section{Simulations}

Consider the system (1a)-(1b) with the following matrices: $A=\left[\begin{array}{cccc}0.9 & 0 & 0 & 0 \\ 0 & 1 & 0 & 0 \\ 0 & 0 & \cos (\psi) & -\sin (\psi) \\ 0 & 0 & \sin (\psi) & \cos (\psi)\end{array}\right], B=\left[\begin{array}{l}0 \\ 1 \\ 0 \\ 1\end{array}\right]$, and $C=I$, where $\psi=\frac{\pi}{2}$. The orthogonal part of the state is 3 dimensional, and the controllability index of the orthogonal part is $\kappa=3$.

Example 1. The simulation data was chosen to be: $x_{0} \sim$ $\mathcal{N}(0, I), w_{t} \sim \mathcal{N}(0,10 I), v_{t}=\mathcal{N}(0,10 I), Q=I, R=1$, $N=5, N_{c}=\kappa=3$, and $\varphi$ the usual piecewise linear saturation function with $\varphi_{\max }=1$. For this example the theoretical bound on the input is $U_{\max } \tilde{=} 453$ for a choice of $\varepsilon=10$.

We simulated the system above using Algorithm 1 for the discrete-time interval $[0,100]$. In comparison, we simulated also the policy (38) proposed by the authors in [39] whenever the state is estimated using a Kalman filter, with the understanding that this goes beyond the results in [39], since [39] deals only with the case of perfect state information. We also simulated the standard LQG controller for this system with post-saturation of the obtained controls. The average state norm as well as the standard deviation of the state norm using the three strategies are depicted in Figure 1 and the total costs are plotted in Figure 2. Figure 2 shows approximately $16 \%$ 
improvement in the cost after 100 time steps by using Algorithm 1 versus the policy (38) in [39] coupled with a Kalman filter. The performance of our policy is close to that of clipped LQG, which remains mean-square 'optimal' since the theoretical upper bound on the control authority is never reached by the LQG policy, i.e., the LQG policy is never clipped (for our choice of initial condition $x_{0}$ ). The optimization problem was solved using yalmip [31] and sdpt3 [42] and the solver times were as follows: minimum $=0.1238$ second, maximum $=0.5831$ second, average $=0.1691$ second, and standard deviation $=0.0144$ second. This data pertains to an Intel(R) Core(TM)2 Duo CPU running at $2.66 \mathrm{GHz}$, with a Linux operating system. The computation of the matrices $\Lambda^{e \varphi}\left(P^{\circ}\right), \Lambda^{\varphi}\left(P^{\circ}\right), \Lambda^{w \varphi}\left(P^{\circ}\right)$, and $\Lambda^{\varphi \varphi}\left(P^{\circ}\right)$ was done off-line using the steady state error covariance matrix $P^{\circ}$, as discussed in the previous section, via classical Monte Carlo integration using $10^{5}$ samples.

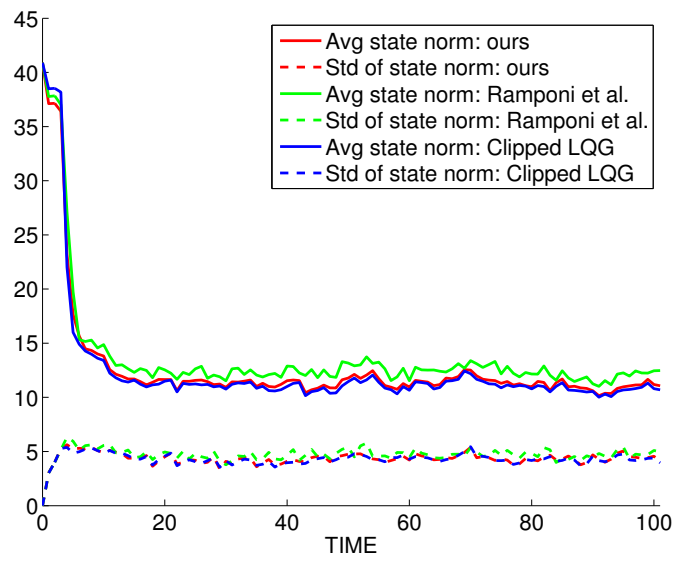

Fig. 1. Average and standard deviation of the state norm for $U_{\max }=453$

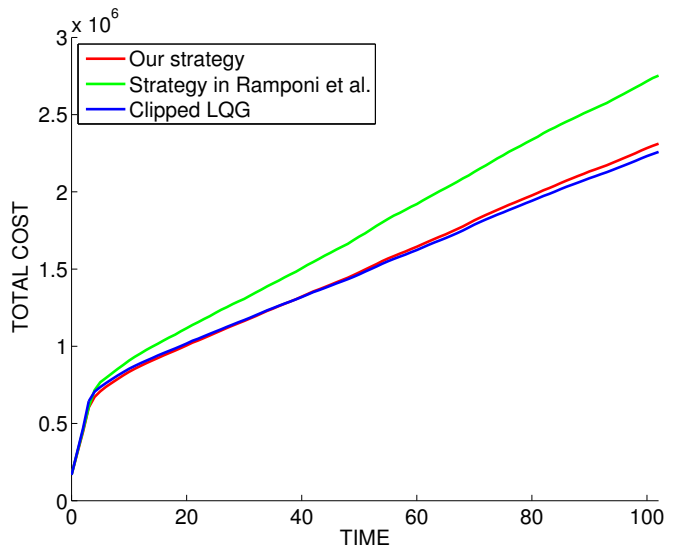

Fig. 2. Total cost for $U_{\max }=453$

Example 2. We also simulated the same system as before with $Q=100 I$ and $R=1$. In this case the theoretical bound $\zeta=336.6$ and the corresponding $U_{\max }=440.45$.
As this theoretical bound on $\zeta$ is conservative, we reduced $\zeta$ down to 2 and chose $\varepsilon=0.5$, which result in $U_{\max }=3.2664$. This choice of $\zeta$ is far below the required theoretical bound (which was given in Example 1 above) and as such the stability guarantees in the article do not apply anymore, i.e., there is no theoretical guarantee that the closed-loop system is mean-square bounded. However, it is apparent from Figures 3 and 4 that our policy is stabilizing. It is important to notice that the clipped LQG policy hits the saturation level $U_{\max }$ quite often, whereas our policy does not, as seen in Figure 5. However, despite the fact that our strategy does not take full advantage of the available control authority, it is still able to outperform the clipped LQG as well as the adapted policy in [39].

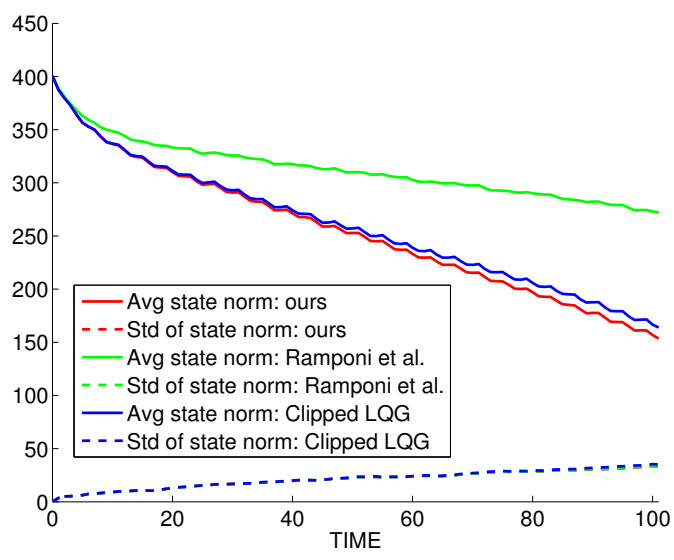

Fig. 3. Average and standard deviation of the state norm for $U_{\max }=3.2664$

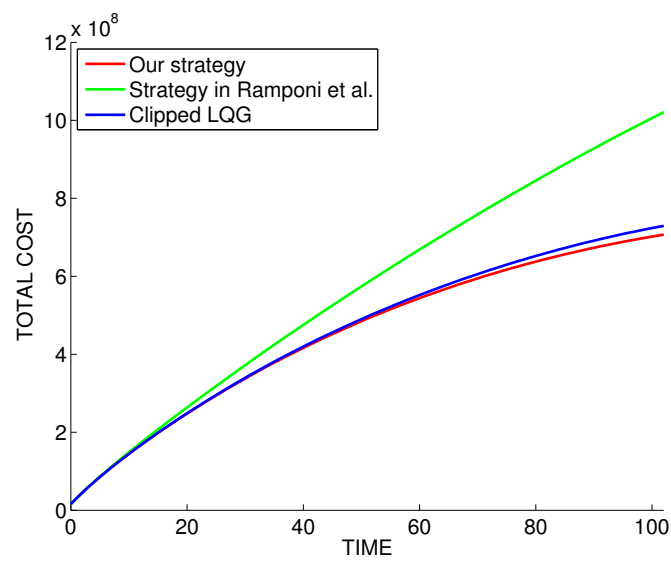

Fig. 4. Total cost for $U_{\max }=3.2664$

\section{Conclusions}

We presented a method for stochastic receding horizon control of discrete-time linear systems with process and measurement noise and bounded input policies. We showed that the optimization problem solved periodically is successively feasible and convex. Moreover, we illustrated how a certain stability condition can be utilized 


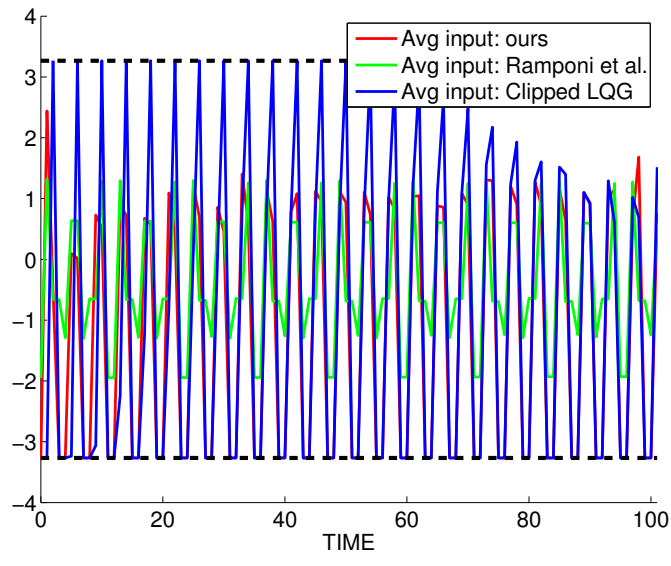

Fig. 5. Average inputs for $U_{\max }=3.2664$

to ensure that the application of the receding horizon controller renders the state of the system mean-square bounded. We discussed how certain matrices in the cost function can be computed off-line and provided examples that illustrate our approach, showing conditions under which it outperforms certain competing approaches.

\section{Acknowledgements}

This research was partially supported by the Swiss National Science Foundation under grant 200021-122072 and by the European Commission under the projects Feednetback FP7-ICT-223866 (www.feednetback.eu) and MoVeS FP7-ICT-2009-257005.

\section{References}

[1] I. Batina. Model predictive control for stochastic systems by randomized algorithms. $\mathrm{PhD}$ thesis, Technische Universiteit Eindhoven, 2004.

[2] A. Ben-Tal, S. Boyd, and A. Nemirovski. Extending scope of robust optimization: Comprehensive robust counterparts of uncertain problems. Journal of Mathematical Programming, 107:63-89, 2006.

[3] A. Ben-Tal, A. Goryashko, E. Guslitzer, and A. Nemirovski. Adjustable robust solutions of uncertain linear programs. Mathematical Programming, 99(2):351-376, 2004.

[4] A. Ben-Tal and A. Nemirovski. Lectures on Modern Convex Optimization: Analysis, Algorithms, and Engineering Practice. SIAM, 2001.

[5] D. Bernardini and A. Bemporad. Scenario-based model predictive control of stochastic constrained linear systems. In IEEE Conference on Decision and Control, pages 63336338, Shanghai, China, 2009.

[6] D. S. Bernstein. Matrix Mathematics. Princeton University Press, 2 edition, 2009.

[7] D. P. Bertsekas. Dynamic Programming and Optimal Control, volume 1. Athena Scientific, 2 edition, 2000.

[8] D. P. Bertsekas. Dynamic Programming and Optimal Control, volume 2. Athena Scientific, 3 edition, 2007.

[9] D. Bertsimas and D. B. Brown. Constrained stochastic LQC: a tractable approach. IEEE Transactions on Automatic Control, 52(10):1826-1841, 2007.
[10] D. Bertsimas, D. A. Iancu, and P. A. Parrilo. Optimality of affine policies in multi-stage robust optimization. Mathematics of Operations Research, 35(2):363-394, May 2010.

[11] L. Blackmore. A probabilistic particle control approach to optimal, robust predictive control. In Proceedings of the AIAA Guidance, Navigation and Control Conference, Aug. 2006.

[12] S. Boyd and L. Vandenberghe. Convex Optimization. Cambridge University Press, Cambridge, 2004. Sixth printing with corrections, 2008.

[13] M. Cannon, Q. Cheng, B. Kouvaritakis, and S.V. Raković. Stochastic tube MPC with state estimation. In Proc. of the 19th International Symposium on Mathematical Theory of Networks and Systems - MTNS 2010, pages 11-16, July 2010.

[14] M. Cannon, B. Kouvaritakis, and X. Wu. Model predictive control for systems with stochastic multiplicative uncertainty and probabilistic constraints. Automatica, 45(1):167-172, Jan. 2009.

[15] M. Cannon, B. Kouvaritakis, and X. Wu. Probabilistic constrained MPC for systems with multiplicative and additive stochastic uncertainty. IEEE Transactions on Automatic Control, 54(7):1626-1632, July 2009.

[16] D. Chatterjee, P. Hokayem, and J. Lygeros. Stochastic receding horizon control with bounded control inputs: a vector space approach. IEEE Transactions on Automatic Control, 2009. To appear. http://arxiv.org/abs/0903. 5444

[17] E. Cinquemani, M. Agarwal, D. Chatterjee, and J. Lygeros. Convexity and convex approximations of discrete-time stochastic control problems with constraints. Automatica, To appear, 2011. http://arxiv.org/abs/0905.3447

[18] P. D. Couchman, M. Cannon, and B. Kouvaritakis. Stochastic MPC with inequality stability constraints. Automatica, 42(12):2169-2174, 2006.

[19] P. J. Goulart, E. C. Kerrigan, and J. M. Maciejowski. Optimization over state feedback policies for robust control with constraints. Automatica, 42(4):523-533, 2006.

[20] P. Hokayem, D. Chatterjee, and J. Lygeros. On stochastic model predictive control with bounded control inputs. In Proceedings of the combined 48th IEEE Conference on Decision 8 Control and 28th Chinese Control Conference, pages 6359-6364, 2009. http://arxiv.org/abs/0902.3944.

[21] P. Hokayem, D. Chatterjee, F. Ramponi, G. Chaloulos, and J. Lygeros. Stable stochastic receding horizon control of linear systems with bounded control inputs. In Int. Symposium on Mathematical Theory of Networks and Systems (MTNS), pages 31-36, Budapest, Hungary, July 2010.

[22] P. Hokayem, E. Cinquemani, D. Chatterjee, and J. Lygeros. Stochastic MPC with imperfect state information and bounded controls. In Proceedings of the UKACC Int. Conference on Control, Coventry, UK, Sept. 2010.

[23] R. A. Horn and C. R. Johnson. Matrix Analysis. Cambridge University Press, Cambridge, 1990.

[24] A. H. Jazwinski. Stochastic Processes and Filtering Theory. Academic Press, 1970.

[25] E. W. Kamen and J. K. Su. Introduction to Optimal Estimation. Springer, London, UK, 1999.

[26] W. K. Klein Haneveld. On integrated chance constraints. In Stochastic programming (Gargnano), volume 76 of Lecture Notes in Control and Inform. Sci., pages 194-209. Springer, Berlin, 1983. 
[27] W. K. Klein Haneveld and M. H. van der Vlerk. Integrated chance constraints: reduced forms and an algorithm. Computational Management Science, 3(4):245-269, 2006.

[28] P. R. Kumar and P. Varaiya. Stochastic Systems: Estimation, Identification, and Adaptive Control. Prentice Hall, 1986.

[29] P. Li, M. Wendt, and G. Wozny. A probabilistically constrained model predictive controller. Automatica, 38(7):1171-1176, July 2002.

[30] J. Löfberg. Minimax Approaches to Robust Model Predictive Control. PhD thesis, Linköpings Universitet, 2003.

[31] J. Löfberg. YALMIP : A Toolbox for Modeling and Optimization in MATLAB. In Proceedings of the CACSD Conference, Taipei, Taiwan, 2004.

[32] J. M. Maciejowski. Predictive Control with Constraints. Prentice Hall, 2001.

[33] M. Maciejowski, A. Lecchini, and J. Lygeros. NMPC for complex stochastic systems using Markov Chain Monte Carlo. In Assessment and Future Directions of Nonlinear Model Predictive Control, volume 358 of Lecture Notes in Control and Information Sciences, pages 269-281, Stuttgart, Germany, 2007. Springer.

[34] L. Magni, D. Pala, and R. Scattolini. Stochastic model predictive control of constrained linear systems with additive uncertainty. In European Control Conference, pages 22352240, Budapest, Hungary, 2009.

[35] D. Q. Mayne, J. B. Rawlings, C. V. Rao, and P. O. M. Scokaert. Constrained model predictive control: stability and optimality. Automatica, 36(6):789-814, Jun 2000.

[36] F. Oldewurtel, C.N. Jones, and M. Morari. A tractable approximation of chance constrained stochastic MPC based on affine disturbance feedback. In Proceedings of the IEEE Conference on Decision and Control, Cancun, Mexico, Dec 2008.

[37] R. Pemantle and J. S. Rosenthal. Moment conditions for a sequence with negative drift to be uniformly bounded in $L^{r}$. Stochastic Processes and their Applications, 82(1):143-155, 1999.

[38] J. A. Primbs and C. H. Sung. Stochastic receding horizon control of constrained linear systems with state and control multiplicative noise. IEEE Transactions on Automatic Control, 54(2):221-230, Feb. 2009.

[39] F. Ramponi, D. Chatterjee, A. Milias-Argeitis, P. Hokayem, and J. Lygeros. Attaining mean square boundedness of a marginally stable stochastic linear system with a bounded control input. IEEE Transactions on Automatic Control, 55(10):2414-2418, 2010.

[40] A. Schwarm and M. Nikolaou. Chance-constrained model predictive control. AlChE Journal, 45(8):1743-1752, 1999.

[41] J. Skaf and S. Boyd. Design of affine controllers via convex optimization. IEEE Transactions on Automatic Control, 55(11):2476-2487, Oct. 2010.

[42] K.C. Toh, M.J. Todd, and R.H. Tatuncu. SDPT3- a Matlab software package for semidefinite programming. Optimization Methods and Software, (11):545-581, 1999. http://www. math.nus.edu.sg/ mattohkc/sdpt3.html

[43] D. H. van Hessem and O. H. Bosgra. A full solution to the constrained stochastic closed-loop MPC problem via state and innovations feedback and its receding horizon implementation. In Proceedings of the 42nd IEEE Conference on Decision and Control, volume 1, pages 929-934, 2003.

[44] Y. Wang and S. Boyd. Peformance bounds for linear stochastic control. Systems and Control Letters, 58(3):178$182,2009$.
[45] J. Yan and R. R. Bitmead. Incorporating state estimation into model predictive control and its application to network traffic control. Automatica, 41:595-604, 2005.

[46] Y. D. Yang, E. D. Sontag, and H. J. Sussmann. Global stabilization of linear discrete-time systems with bounded feedback. Systems and Control Letters, 30(5):273-281, 1997.

\section{Appendix: Proofs}

We begin by considering the estimation equation in (7) and combining it with (9) and the system dynamics (1a)-(1b) to obtain (recall that $\hat{x}_{t}$ is used instead of $\hat{x}_{t \mid t}$ for notational simplicity) $\hat{x}_{t+1}=$ $A \hat{x}_{t}+B u_{t}+K_{t}\left(C A\left(x_{t}-\hat{x}_{t}\right)+C w_{t}+v_{t+1}\right)$, where $K_{t}=\left(A P_{t \mid t} A^{\top}+\Sigma_{w}\right) C^{\top}\left(C\left(A P_{t \mid t} A^{\top}+\Sigma_{w}\right) C^{\top}+\Sigma_{v}\right)^{-1}$ and $P_{t \mid t}$ is the error covariance matrix defined in (8). Both $K_{t}$ and $P_{t}$ are uniformly norm-bounded as shown in the following Lemma:

Lemma 8 Consider the system (1a)-(1b), and let Assumption 1-(iv) hold. In addition, assume that $P_{0 \mid 0} \geqslant 0$. Then, there exists a time $T^{\prime} \in \mathbb{N}$ and constants $\rho, \rho_{m}>0$ such that $\operatorname{tr}\left(P_{t \mid t}\right) \leqslant \rho$ and $\left\|K_{t}\right\| \leqslant \rho_{m}, \forall t \geqslant T^{\prime}$.

PROOF. First, observe that $\sum_{i=0}^{\kappa_{1}-1} A^{i} \Sigma_{w}\left(A^{i}\right)^{\top}=$

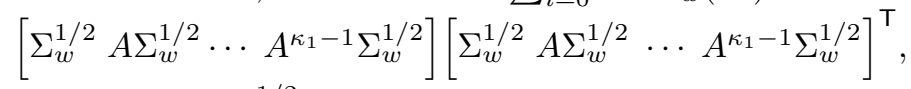
and since $\left(A, \Sigma_{w}^{1 / 2}\right)$ is controllable by Assumption 4(iv), we see that there exists $\kappa_{1} \in \mathbb{N}_{+}$such that for all $k \geqslant \kappa_{1}$ the rank of $\left[\begin{array}{llll}\Sigma_{w}^{1 / 2} & A \Sigma_{w}^{1 / 2} & \cdots & A^{\kappa_{1}-1} \Sigma_{w}^{1 / 2}\end{array}\right]=n$; indeed, $\kappa_{1}$ is the reachability index of $\left(A, \Sigma_{w}^{1 / 2}\right)$. Thus, $\sum_{i=0}^{\kappa_{1}-1} A^{i} \Sigma_{w}\left(A^{i}\right)^{\top}$ is positive definite, and therefore, there exists some $\delta_{1}^{\prime}, \delta_{2}^{\prime}>0$ such that $\delta_{1}^{\prime} I \leqslant \sum_{i=0}^{\kappa_{1}-1} A^{i} \Sigma_{w}\left(A^{i}\right)^{\top} \leqslant \delta_{2}^{\prime} I$. Second, observe that $\sum_{i=0}^{\kappa_{2}-1}\left(A^{i}\right)^{\top} C^{\top} \Sigma_{v}^{-1} C A^{i}=\left[\begin{array}{c}C \\ C A \\ \vdots \\ C A^{\kappa_{2}-1}\end{array}\right]^{\top}\left(I_{\kappa_{2}} \otimes\right.$ $\left.\Sigma_{v}^{-1}\right)\left[\begin{array}{c}C \\ C A \\ \vdots \\ C A^{\kappa_{2}-1}\end{array}\right]$, where $\otimes$ denotes the standard Kronecker product. Since $(A, C)$ is observable by assumption, there exists $\kappa_{2} \in \mathbb{N}_{+}$such that the rank of the matrix $\left[C^{\top} A^{\top} C^{\top} \cdots\left(A^{\kappa_{2}-1}\right)^{\top} C^{\top}\right]^{\top}$ is $n$. The matrix $I_{\kappa_{2}} \otimes \Sigma_{v}^{-1}$ is clearly positive definite by Assumption 4 -(iv), and therefore, we see that there exists $\delta_{1}^{\prime \prime}, \delta_{2}^{\prime \prime}>0$ such that $\delta_{1}^{\prime \prime} I \leqslant \sum_{i=0}^{\kappa_{2}-1}\left(A^{i}\right)^{\top} C^{\top} \Sigma_{v}^{-1} C A^{i} \leqslant \delta_{2}^{\prime \prime} I$. Third, the conditions of Lemma 7.1 in [24, pp. 234] are satisfied, for a choice of $\delta_{1}=\min \left\{\delta_{1}^{\prime}, \delta_{1}^{\prime \prime}\right\}$ and $\delta_{2}=\max \left\{\delta_{2}^{\prime}, \delta_{2}^{\prime \prime}\right\}$, and the bound $P_{t \mid t} \leqslant \rho^{\prime} I$ for some $\rho^{\prime}>0$ is established for all $t \geqslant T^{\prime}:=\max \left\{\kappa_{1}, \kappa_{2}\right\}$. The assertion now follows immediately from: $\mathbb{E}_{\mathcal{Y}_{t}}\left[\left\|x_{t}-\hat{x}_{t}\right\|^{2}\right]=\operatorname{tr}\left(P_{t \mid t}\right) \leqslant$ $n \lambda_{\max }\left(P_{t \mid t}\right) \leqslant n \rho^{\prime}=: \rho$. Since by assumption $\Sigma_{v}>0$, one possible bound on $\left(\left\|K_{t}\right\|\right)_{t \geqslant T^{\prime}}$ is given by $\left\|K_{t}\right\| \leqslant$ $\frac{\left\|A P_{t \mid t} A^{\top}+\Sigma_{w}\right\|\left\|C^{\top}\right\|}{\lambda_{\min }\left(\Sigma_{v}\right)} \leqslant \frac{\left(\left\|\Sigma_{w}\right\|+\|A\|^{2}\left\|P_{t \mid t}\right\|\right)\left\|C^{\top}\right\|}{\lambda_{\min }\left(\Sigma_{v}\right)}=: \rho_{m}$. 
Using the bounds in Lemma 8, we can proceed to prove Proposition 3.

PROOF. [Proof of Proposition 3] Recall the expression of $\Xi_{t}$ in (20) and define the following quantities: $F_{t}:=\left[\begin{array}{llll}A^{N_{c}-1} K_{t} C A & A^{N_{c}-2} K_{t+1} C A & \cdots & K_{t+N_{c}-1} C A\end{array}\right]$, $G_{t}:=\left[A^{N_{c}-1} K_{t} C A^{N_{c}-2} K_{t+1} C \cdots K_{t+N_{c}-1} C\right]$, $H_{t}:=\left[\begin{array}{llll}A^{N_{c}-1} K_{t} & A^{N_{c}-2} K_{t+1} & \cdots & K_{t+N_{c}-1}\end{array}\right]$ Using Lemma 8, we have that $\left\|F_{t}\right\| \leqslant N_{c}\|C A\|\left\|K_{t}\right\| \leqslant$ $N_{c} \rho_{m}\|C A\|,\left\|G_{t}\right\| \leqslant N_{c}\|C\|\left\|K_{\ell}\right\| \leqslant N_{c} \rho_{m}\|C\|$, and $\left\|H_{t}\right\| \leqslant N_{c}\left\|K_{\ell}\right\| \leqslant N_{c} \rho_{m}$, for all $t \geqslant T^{\prime}$. It follows that $\mathbb{E}_{\mathcal{Y}_{t}}\left[\left\|\Xi_{t}\right\|\right] \leqslant N_{c} \rho_{m}\|C A\| \mathbb{E}_{\mathcal{Y}_{t}}\left[\left\|\begin{array}{c}e_{t} \\ \vdots \\ e_{t+N_{c}-1}\end{array}\right\|\right]+$ $N_{c} \rho_{m}\|C\| \mathbb{E}_{\mathcal{Y}_{t}}\left[\left\|\begin{array}{c}w_{t} \\ \vdots \\ w_{t+N_{c}-1}\end{array}\right\|\right]+N_{c} \rho_{m} \mathbb{E}_{\mathcal{Y}_{t}}\left[\left\|\begin{array}{c}v_{t+1} \\ \vdots \\ v_{t+N_{c}}\end{array}\right\|\right]$ $\leqslant N_{c}^{3 / 2} \rho_{m}\left(\|C A\| \sqrt{\rho}+\|C\| \sqrt{\operatorname{tr}\left(\Sigma_{w}\right)}+\sqrt{\operatorname{tr}\left(\Sigma_{v}\right)}\right)=: \zeta$, for all $t \geqslant T^{\prime}$.

Proof of Theorem 5 We begin by showing the first claim in Theorem 5 .

Lemma 9 Consider the system (1a)-(1b), and suppose that Assumption 4 holds. Then assertion (i) of Theorem 5 holds.

PROOF. Convexity: It is clear that $X_{t}^{\top} \mathcal{Q} X_{t}+U_{t}^{\top} \mathcal{R} U_{t}$ is convex in $X_{t}$ and $U_{t}$, and both $X_{t}$ and $U_{t}$ are affine functions of the design parameters $\left(\boldsymbol{\eta}_{t}, \boldsymbol{\Theta}_{t}\right)$ for every realization of the noise sequences $\left(w_{t}\right)_{t \in \mathbb{N}}$ and $\left(v_{t}\right)_{t \in \mathbb{N}}$. Since taking expectation of a convex function retains convexity [12], we conclude that the cost $V_{t}=\mathbb{E}_{\mathcal{Y}_{t}}\left[X_{t}^{\top} \mathcal{Q} X_{t}+U_{t}^{\top} \mathcal{R} U_{t}\right]$ is convex in $\left(\boldsymbol{\eta}_{t}, \boldsymbol{\Theta}_{t}\right)$. Similarly, the constraints (17) and (22) are convex in $\left(\boldsymbol{\eta}_{t}, \boldsymbol{\Theta}_{t}\right)$ as they are a composition of convex and affine functions [12].

SOCP Formulation: Substituting the augmented dynamics (5) into the objective function (6), we have that $\mathcal{J}_{t}=\mathbb{E}_{\mathcal{Y}_{t}}\left[\left\|\mathcal{A} x_{t}+\mathcal{B} U_{t}+\mathcal{D} W_{t}\right\|_{\mathcal{Q}}^{2}+\left\|U_{t}\right\|_{\mathcal{R}}^{2}\right]=$ $\mathbb{E}_{\mathcal{Y}_{t}}\left[\left\|U_{t}\right\|_{\mathcal{M}}^{2}+2 U_{t}^{\top} \mathcal{B}^{\top} \mathcal{Q}\left(\mathcal{A} x_{t}+\mathcal{D} W_{t}\right)\right]+\mathbb{E}_{\mathcal{Y}_{t}}\left[\left\|W_{t}\right\|_{\mathcal{D}^{\top} \mathcal{Q D}}^{2}+\right.$ $\left.\left\|x_{t}\right\|_{\mathcal{A}^{\top} \mathcal{Q \mathcal { A }}}^{2}\right]$, where we have used the fact that the noise $W_{t}$ is zero-mean (and $\mathcal{M}=\mathcal{R}+\mathcal{B}^{\top} \mathcal{Q B}$ ). Note that the last term above does not depend on the decision variables so we shall henceforth drop it from the optimization. Now, substituting the policy (15) into the last equation and completing the square yields $\mathcal{J}_{t} \cong \mathbb{E}_{\mathcal{Y}_{t}}\left[\left(\boldsymbol{\eta}_{t}+\right.\right.$ $\left.\boldsymbol{\Theta}_{t} \varphi\left(Y_{t}-\hat{Y}_{t}\right)\right)^{\top} \mathcal{M}\left(\boldsymbol{\eta}_{t}+\boldsymbol{\Theta}_{t} \varphi\left(Y_{t}-\hat{Y}_{t}\right)\right)+2\left(\boldsymbol{\eta}_{t}+\right.$ $\left.\left.\boldsymbol{\Theta}_{t} \varphi\left(Y_{t}-\hat{Y}_{t}\right)\right)^{\top} \mathcal{B}^{\top} \mathcal{Q}\left(\mathcal{A} x_{t}+\mathcal{D} W_{t}\right)\right]\left\|\boldsymbol{\eta}_{t}+\boldsymbol{\Theta}_{t} \Lambda_{t}^{\varphi}\right\|_{\mathcal{M}}^{2}+$ $\operatorname{tr}\left(\boldsymbol{\Theta}_{t}^{\top} \mathcal{M} \Theta_{t}\left(\Lambda_{t}^{\varphi \varphi}-\Lambda_{t}^{\varphi} \Lambda_{t}^{\varphi \top}\right)\right)+2 \boldsymbol{\eta}_{t}^{\top} \mathcal{B}^{\top} \mathcal{Q} \mathcal{A} \hat{x}_{t}$ $+2 \operatorname{tr}\left(\Theta_{t}^{\top} \mathcal{B}^{\top} \mathcal{Q}\left(\mathcal{A} \Lambda_{t}^{x \varphi}+\mathcal{D} \Lambda_{t}^{w \varphi}\right)\right)$. Note that $\Lambda_{t}^{\varphi \varphi}-$ $\Lambda_{t}^{\varphi} \Lambda_{t}^{\varphi \top} \geqslant 0$, so we can further write the quadratic term in $\boldsymbol{\Theta}_{t}$ in a symmetric fashion, i.e., since $\Lambda_{t}^{\varphi \varphi}-$ $\Lambda_{t}^{\varphi} \Lambda_{t}^{\varphi \mathrm{T}}$ is positive semidefinite, there exists a ma$\operatorname{trix} L$ so that $\Lambda_{t}^{\varphi \varphi}-\Lambda_{t}^{\varphi} \Lambda_{t}^{\varphi \top}=L L^{\top}$. It follows that $\operatorname{tr}\left(\Theta_{t}^{\top} \mathcal{M} \Theta_{t}\left(\Lambda_{t}^{\varphi \varphi}-\Lambda_{t}^{\varphi} \Lambda_{t}^{\varphi \top}\right)\right)=\operatorname{tr}\left(\Theta_{t}^{\top} \mathcal{M} \Theta_{t} L L^{\top}\right)=$ $\operatorname{tr}\left(L^{\top} \boldsymbol{\Theta}_{t}^{\top} \mathcal{M} \Theta_{t} L\right)$. Using the epigraph formulation gives the cost (24) and constraint (25).

Concerning the constraint (26), we have shown in $[20,16]$ that combining the constraint $\left\|u_{t}\right\|_{\infty} \leqslant U_{\max }$ and the class of policies (15) is equivalent to the constraints $\left|\left(\boldsymbol{\eta}_{t}\right)_{i}\right|+\left\|\left(\boldsymbol{\Theta}_{t}\right)_{i}\right\|_{1} \varphi_{\max } \leqslant U_{\max }$ for all $i=1, \ldots, N m$. Substituting (15) into the stability constraint (22), we obtain

$$
\begin{aligned}
\| A_{o}^{N_{c}} \hat{x}_{t}^{o} & +\mathfrak{R}_{N_{c}}\left(A_{o}, B_{o}\right)\left(\boldsymbol{\eta}_{t}\right)_{1: N_{c} m} \\
+ & \mathfrak{R}_{N_{c}}\left(A_{o}, B_{o}\right)\left(\boldsymbol{\Theta}_{t}\right)_{1: N_{c} m} \varphi\left(Y_{t}-\hat{Y}_{t}\right) \| \\
\leqslant & \left\|A_{o}^{N_{c}} \hat{x}_{t}^{o}+\mathfrak{R}_{N_{c}}\left(A_{o}, B_{o}\right)\left(\boldsymbol{\eta}_{t}\right)_{1: N_{c} m}\right\| \\
& +\left\|\Re_{N_{c}}\left(A_{o}, B_{o}\right)\left(\boldsymbol{\Theta}_{t}\right)_{1: N_{c} m} \varphi\left(Y_{t}-\hat{Y}_{t}\right)\right\| \\
\leqslant & \left\|A_{o}^{N_{c}} \hat{x}_{t}^{o}+\mathfrak{R}_{N_{c}}\left(A_{o}, B_{o}\right)\left(\boldsymbol{\eta}_{t}\right)_{1: N_{c} m}\right\| \\
& +\sqrt{n_{o}}\left\|\mathfrak{R}_{N_{c}}\left(A_{o}, B_{o}\right)\left(\boldsymbol{\Theta}_{t}\right)_{1: N_{c} m} \varphi\left(Y_{t}-\hat{Y}_{t}\right)\right\|_{\infty} \\
\leqslant & \left\|A_{o}^{N_{c}} \hat{x}_{t}^{o}+\mathfrak{R}_{N_{c}}\left(A_{o}, B_{o}\right)\left(\boldsymbol{\eta}_{t}\right)_{1: N_{c} m}\right\| \\
& +\sqrt{n_{o}}\left\|\mathfrak{R}_{N_{c}}\left(A_{o}, B_{o}\right)\left(\boldsymbol{\Theta}_{t}\right)_{1: N_{c} m}\right\|_{\infty} \varphi_{\max } .
\end{aligned}
$$

Enforcing that the last term above is $\leqslant\left\|\hat{x}_{t}^{o}\right\|-\left(\zeta-\frac{\varepsilon}{2}\right)$, whenever $\left\|\hat{x}_{t}^{o}\right\| \geqslant \zeta+\varepsilon$, is equivalent to the constraint (27), where the decision variables are now $\left(z_{2}, z_{3}, \boldsymbol{\eta}_{t}, \boldsymbol{\Theta}_{t}\right)$. Moreover, if the constraint (27) is satisfied, then the stability constraint (22) is satisfied as well. As such, the optimization problem solved in Theorem 5-(i) is a conservative approximation of (23) due to the fact that the constraint (27) is tighter than (22). Feasibility: It remains to show that all the constraints are simultaneously feasible. Inspired by the work in [39], we consider the candidate controller

$$
\tilde{u}_{t, t+N_{c}-1}=\left[\begin{array}{c}
\eta_{t} \\
\vdots \\
\eta_{t+N_{c}-1}
\end{array}\right]=-\mathfrak{R}_{N_{c}}\left(A_{o}, B_{o}\right)^{\dagger} \operatorname{sat}_{r}\left(A_{o}^{N_{c}} \hat{x}_{t}^{o}\right)
$$

with $\eta_{t+N_{c}}=\eta_{t+N_{c}+1}=\cdots=\eta_{t+N-1}=0$ and $\boldsymbol{\Theta}_{t}=0$, where $r:=\zeta+\varepsilon / 2$ and recall that $\operatorname{sat}_{r}(v)=v$ if $\|v\| \leqslant r$ and $\operatorname{sat}_{r}(v)=r v /\|v\|$ if $\|v\|>r$. First, we have that $\left\|\tilde{u}_{t, t+N_{c}-1}\right\|_{\infty} \leqslant\left\|\tilde{u}_{t, t+N_{c}-1}\right\|_{2} \leqslant$ $\sigma_{\min }\left(\Re_{N_{c}}\left(A_{o}, B_{o}\right)\right)^{-1}(\zeta+\varepsilon / 2)=U_{\text {max }}^{*}$, and the constraint (2) is feasible. Regarding the constraint (22), we have that $\left\|A_{o}^{N_{c}} \hat{x}_{t}^{o}+\mathfrak{R}_{N_{c}}\left(A_{o}, B_{o}\right) \tilde{u}_{t, t+N_{c}-1}\right\|=$ $\left\|\hat{x}_{t}^{o}\right\|-r \leqslant\left\|\hat{x}_{t}^{o}\right\|-(\zeta+\varepsilon / 2)$, whenever $\left\|\hat{x}_{t}^{o}\right\| \geqslant \zeta+\varepsilon$, where the first equality follows from the orthogonality of $A_{o}$ (see [39]), and the constraint (22) is also feasible. Finally, it is easy to see that the cost (24) is linear and (25) through (27) are second-order cone constraints, hence the optimization program is a SOCP [4].

The following result pertains to mean-square boundedness of the Schur subsystem $\hat{x}_{t}^{s}$ of the estimator, i.e., 
$\hat{x}_{t+1}^{s}=A_{s} \hat{x}_{t}^{s}+B_{s} u_{t}+\left(K_{t}\right)_{1: n_{s}}\left(C A\left(x_{t}-\hat{x}_{t}\right)+C w_{t}+\right.$ $\left.v_{t+1}\right)$, where $\left(K_{t}\right)_{1: n_{s}}$ are the first $n_{s}$ rows of the gain $K_{t}$.

Lemma 10 Let Assumption 1 hold. Then there exists a constant $\gamma_{s}>0$, depending on the given problem parameters, such that $\mathbb{E}_{\mathcal{Y}_{T^{\prime}}}\left[\left\|\hat{x}_{t}^{s}\right\|^{2}\right] \leqslant \gamma_{s}, \forall t \geqslant T^{\prime}$, where $T^{\prime}$ is as defined in Proposition 3.

PROOF. Since the matrix $A_{s}$ is Schur stable, there exists a positive definite matrix $M \in \mathbb{R}^{n_{s} \times n_{s}}$ that satisfies $A_{s}^{\top} M A_{s}-M=-I$, see [6, Proposition 11.10.5]. Pick a constant $\nu \in] 0, \min \left\{1,1 / \lambda_{\max }(M)\right\}[$ such that $A_{s}^{\top} M A_{s}-M \leqslant-\nu M$. Then for any $t \in \mathbb{N}, \mathbb{E}_{\mathcal{Y}_{t}}\left[\left\|\hat{x}_{t+1}^{s}\right\|_{M}^{2}\right]-\left\|\hat{x}_{t}^{s}\right\|_{M}^{2} \leqslant-\nu\left\|\hat{x}_{t}^{s}\right\|_{M}^{2}+$ $2 \mathbb{E}_{\mathcal{Y}_{t}}\left[\left(\hat{x}_{t}^{s}\right)^{\top} A_{s}^{\top} M B_{s} u_{t}\right]+$

$\mathbb{E}_{\mathcal{Y}_{t}}\left[\left\|\left(K_{t}\right)_{1: n_{s}}\left(C A\left(x_{t}-\hat{x}_{t}\right)+C w_{t}+v_{t+1}\right)\right\|_{M}^{2}\right]$, where $\|x\|_{M}^{2}:=x^{\top} M x$. Using Young's inequality and Assumption 1 , we have that $2 \mathbb{E}_{\mathcal{Y}_{t}}\left[\left(\hat{x}_{t}^{s}\right)^{\top} A_{s}^{\top} M B_{s} u_{t}\right] \leqslant$ $\varepsilon \mathbb{E}_{\mathcal{Y}_{t}}\left[\left\|A_{s} \hat{x}_{t}^{s}\right\|_{M}^{2}\right]+\frac{1}{\varepsilon} \mathbb{E}_{\mathcal{Y}_{t}}\left[\left\|B_{s} u_{t}\right\|_{M}^{2}\right] \leqslant \varepsilon \mathbb{E}_{\mathcal{Y}_{t}}\left[\left\|A_{s} \hat{x}_{t}^{s}\right\|_{M}^{2}\right]+$ $\frac{m \sigma_{\max }\left(B_{s}^{T} M B_{s}\right)}{\varepsilon} U_{\max }^{2}$, for $\varepsilon>0$ and for all $t \in \mathbb{N}$. Also, using Lemma 8 and Assumption 1, we have that $\mathbb{E}_{\mathcal{Y}_{t}}\left[\left\|\left(K_{t}\right)_{1: n_{s}}\left(C A\left(x_{t}-\hat{x}_{t}\right)+C w_{t}+v_{t+1}\right)\right\|_{M}^{2}\right] \leqslant$ $3 \lambda_{\max }(M) \rho_{m}^{2}\left(\|C A\|^{2} \rho+\|C\|^{2} \operatorname{tr}\left(\Sigma_{w}\right)+\operatorname{tr}\left(\Sigma_{v}\right)\right)$. Choose an $\varepsilon \leqslant \frac{\nu}{2\left\|A_{s}\right\|_{M}^{2}}$ and let $c:=\frac{m \sigma_{\max }\left(B_{s}^{T} M B_{s}\right)}{\varepsilon} U_{\max }^{2}+$ $3 \lambda_{\max }(M) \rho_{m}^{2}\left(\|C A\|^{2} \rho+\|C\|^{2} \operatorname{tr}\left(\Sigma_{w}\right)+\operatorname{tr}\left(\Sigma_{v}\right)\right)$, then we have $\mathbb{E}_{\mathcal{Y}_{t}}\left[\left\|\hat{x}_{t+1}^{s}\right\|_{M}^{2}\right] \leqslant\left(1-\frac{\nu}{2}\right)\left\|\hat{x}_{t}^{s}\right\|_{M}^{2}+c$, for all $t \geqslant T^{\prime}$. Iterating the last inequality, we have $\mathbb{E}_{\mathcal{Y}_{T^{\prime}}}\left[\left\|\hat{x}_{t}^{s}\right\|_{M}^{2}\right] \leqslant\left(1-\frac{\nu}{2}\right)^{\left(t-T^{\prime}\right)}\left\|\hat{x}_{T^{\prime}}^{s}\right\|_{M}^{2}+\sum_{i=0}^{t-T^{\prime}-1}\left(1-\frac{\nu}{2}\right)^{i} c$ $\leqslant\left\|\hat{x}_{T^{\prime}}^{s}\right\|_{M}^{2}+\left(1-\frac{\nu}{2}\right)^{-1} c$, for all $t \geqslant T^{\prime}$. Setting $\gamma_{s}:=\left\|\hat{x}_{T^{\prime}}^{s}\right\|_{M}^{2}+\left(1-\frac{\nu}{2}\right)^{-1} c$, completes the proof.

We consider next the orthogonal subsystem of the estimator and show that the process $\left(\hat{x}_{t}^{o}\right)_{t \in \mathbb{N}}$ is mean-square bounded. We shall rely on the following fundamental result pertaining to mean-square boundedness of a general random sequence $\left(\xi_{t}\right)_{t \in \mathbb{N}}$; it is an immediate consequence of [37, Theorem 1].

Proposition 11 Let $\left(\xi_{t}\right)_{t \in \mathbb{N}}$ be a sequence of nonnegative random variables on some probability space $(\Omega, \mathfrak{F}, \mathbb{P})$, and let $\left(\mathfrak{F}_{t}\right)_{t \in \mathbb{N}}$ be any filtration to which $\left(\xi_{t}\right)_{t \in \mathbb{N}}$ is adapted. Suppose that there exist constants $\varepsilon>0$, and $J, M<\infty$, such that $\xi_{0} \leqslant J$, and for all $t \in \mathbb{N}$ :

$$
\begin{aligned}
& \mathbb{E}_{\mathfrak{F}_{t}}\left[\xi_{t+1}-\xi_{t}\right] \leqslant-\frac{\varepsilon}{2} \quad \text { on the event }\left\{\xi_{t}>J\right\} \\
& \text { and } \quad \mathbb{E}_{\xi_{0}, \ldots, \xi_{t}}\left[\left|\xi_{t+1}-\xi_{t}\right|^{4}\right] \leqslant M
\end{aligned}
$$

Then there exists a constant $\gamma=\gamma(\varepsilon, J, M)>0$ such that $\sup _{t \in \mathbb{N}} \mathbb{E}\left[\xi_{t}^{2}\right] \leqslant \gamma$.
Lemma 12 Let Assumptions 1 and 4 hold. Then there exists a constant $\gamma_{o}>0$, depending on the given problem parameters, such that

$$
\mathbb{E}_{\mathcal{Y}_{T}}\left[\left\|\hat{x}_{t}^{o}\right\|^{2}\right] \leqslant \gamma_{o}, \quad \forall t \geqslant T:=N_{c}\left\lceil T^{\prime} / \kappa\right\rceil
$$

PROOF. Consider the subsampled process $\hat{x}_{t+N_{c}}^{o}=A_{o}^{N_{c}} \hat{x}_{t}^{o}+\Re_{N_{c}}\left(A_{o}, B_{o}\right) u_{t, t+N_{c}-1}+\left(\Xi_{t}\right)_{n-n_{o}+1: n}$ for $t=0, N_{c}, 2 N_{c}, \cdots$, where $\Xi_{t}$ is as defined in (20) and $u_{t, t+N_{c}-1}:=\left[\begin{array}{c}u_{t} \\ \vdots \\ u_{t+N_{c}-1}\end{array}\right]$. We shall first verify the two conditions (39) and (40) of Proposition 11 for the pro-

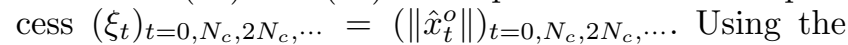
triangle inequality, we have that $\mathbb{E}_{\mathcal{Y}_{t}}\left[\left\|\hat{x}_{t+N_{c}}^{o}\right\|-\left\|\hat{x}_{t}^{o}\right\|\right] \leqslant$ $\mathbb{E}_{\mathcal{Y}_{t}}\left[\left\|A_{o}^{N_{c}} \hat{x}_{t}^{o}+\mathfrak{R}_{N_{c}}\left(A_{o}, B_{o}\right) u_{t, t+N_{c}-1}\right\|-\left\|\hat{x}_{t}^{o}\right\|\right]+$ $\mathbb{E}_{\mathcal{Y}_{t}}\left[\left\|\left(\Xi_{t}\right)_{n-n_{o}+1: n}\right\|\right]$. We know from Proposition 3 that there exists a uniform (with respect to time $t$ ) upper bound $\zeta$ for the last term on the right-hand side of the last inequality for $t \geqslant T:=N_{c}\left\lceil T^{\prime} / \kappa\right\rceil$. Accordingly, we have $\mathbb{E}_{\mathcal{Y}_{t}}\left[\left\|\hat{x}_{t+N_{c}}^{o}\right\|-\left\|\hat{x}_{t}^{o}\right\|\right] \leqslant \mathbb{E}_{\mathcal{Y}_{t}}\left[\| A_{o}^{N_{c}} \hat{x}_{t}^{o}\right.$ $\left.+\mathfrak{R}_{N_{c}}\left(A_{o}, B_{o}\right) u_{t, t+N_{c}-1}\|-\| \hat{x}_{t}^{o} \|\right]+\zeta \leqslant-\frac{\varepsilon}{2}$ whenever $\left\|\hat{x}_{t}^{o}\right\| \geqslant \zeta+\varepsilon$ for all $t=T, T+N_{c}, T+2 N_{c}, \cdots$, where the last inequality follows from the satisfaction of the stability constraint (22); condition (39) of Proposition 11 is satisfied. By orthogonality of $A_{o}$, we have that $\left\|\hat{x}_{t}^{o}\right\|=\left\|A_{o}^{N_{c}} \hat{x}_{t}^{o}\right\|$. It follows by the triangle inequality that, for all $t=T, T+N_{c}, T+2 N_{c}, \cdots$

$\mathbb{E}_{\left\{\left(\left\|\hat{x}_{i}^{o}\right\|\right)_{i=T, T+N_{c}, \cdots, t}\right\}}\left[\left(\left\|\hat{x}_{t+N_{c}^{o}}^{o}\right\|-\left\|\hat{x}_{t}^{o}\right\|\right)^{4}\right]$
$=\mathbb{E}_{\left\{\left(\left\|\hat{x}_{i}^{o}\right\|\right)_{i=T, T+N_{c}, \cdots, t}\right\}}\left[\left(\left\|\hat{x}_{t+N_{c}}^{o}\right\|-\left\|A_{o}^{N_{c}} \hat{x}_{t}^{o}\right\|\right)^{4}\right]$
$\leqslant \quad \mathbb{E}_{\left\{\left(\left\|\hat{x}_{i}^{o}\right\|\right)_{i=T, T+N_{c}, \cdots, t}\right\}}\left[\left(\left\|\Re_{N_{c}}\left(A_{o}, B_{o}\right) u_{t, t+N_{c}-1}\right\|\right.\right.$ $\left.\left.+\left\|\left(\Xi_{t}\right)_{n-n_{o}+1: n}\right\|\right)^{4}\right]$. Using the inequality $(a+b)^{2} \leqslant$ $2 a^{2}+2 b^{2}$, it follows that

$\mathbb{E}_{\left\{\left(\left\|\hat{x}_{i}^{o}\right\|\right)_{i=T, T+N_{c}, \cdots, t}\right\}}\left[\left(\left\|\hat{x}_{t+N_{c}}^{o}\right\|-\left\|\hat{x}_{t}^{o}\right\|\right)^{4}\right]$

$\leqslant 8 \mathbb{E}_{\left\{\left(\left\|\hat{x}_{i}^{o}\right\|\right)_{i=T, T+N_{c}, \cdots, t}\right\}}\left[\left\|\Re_{N_{c}}\left(A_{o}, B_{o}\right) u_{t, t+N_{c}-1}\right\|^{4}\right.$ $\left.+\left\|\left(\Xi_{t}\right)_{n-n_{o}+1: n}\right\|^{4}\right]$. By design $\left\|u_{i}\right\|_{\infty} \leqslant U_{\max }$. Also, $\Xi_{t}$ is independent of $\left(\left\|\hat{x}_{i}^{o}\right\|\right)_{i=T, T+N_{c}, \cdots, t}$, Gaussian, and has its fourth moment bounded. Therefore, there exists a constant $M>0$ such that

$\left.\mathbb{E}_{\left\{\left(\left\|\hat{x}_{i}^{o}\right\|\right)_{i=T, T+N_{c}, \cdots, t}\right\}}\right\}\left\|\Re_{N_{c}}\left(A_{o}, B_{o}\right) u_{t, t+N_{c}-1}\right\|^{4}$
$\left.+\left\|\left(\Xi_{t}\right)_{n-n_{o}+1: n}\right\|^{4}\right] \leqslant M$, for all $t \geqslant T$. The two conditions of Proposition 11 are verified for the sequence $\left(\left\|\hat{x}_{t}^{o}\right\|\right)_{t=T, T+N_{c}, T+2 N_{c}, \cdots .}$ Thus, by Proposition 11, there exists a constant $\gamma_{o}^{\prime}>0$, depending on the given problem parameters, such that $\mathbb{E}_{\mathcal{Y}_{T}}\left[\left\|\hat{x}_{t}^{o}\right\|^{2}\right] \leqslant \gamma_{o}^{\prime}$, for all 
$t=T, T+N_{c}, T+2 N_{c}, \cdots$. Finally, using a standard argument (as in [39]) we can show the existence of another constant $\gamma_{o} \geqslant \gamma_{o}^{\prime}$ such that the condition (41) holds.

PROOF. [Proof of Theorem 5] Claim (i) of Theorem 5 was proved in Lemma 9. It remains to show claim (ii). We start by asserting the following inequality $\mathbb{E}_{\mathcal{Y}_{T}}\left[\left\|x_{t}\right\|^{2}\right] \leqslant 2 \mathbb{E}_{\mathcal{Y}_{T}}\left[\left\|x_{t}-\hat{x}_{t}\right\|^{2}\right]+2 \mathbb{E}_{\mathcal{Y}_{T}}\left[\left\|\hat{x}_{t}\right\|^{2}\right]$, for all $t \geqslant T=N_{c}\left\lceil T^{\prime} / \kappa\right\rceil$. We know from Lemma 8 that $\mathbb{E}_{\mathcal{Y}_{T}}\left[\left\|x_{t}-\hat{x}_{t}\right\|^{2}\right] \leqslant \rho$ for all $t \geqslant T \geqslant T^{\prime}$. We have that $\left\|\hat{x}_{t}\right\|^{2}=\left\|\hat{x}_{t}^{s}\right\|^{2}+\left\|\hat{x}_{t}^{o}\right\|^{2}$, where $\hat{x}^{s}$ and $\hat{x}^{o}$ are states corresponding to the Schur and orthogonal parts of the system, respectively. It then follows that $\mathbb{E}_{\mathcal{Y}_{T}}\left[\left\|\hat{x}_{t}\right\|^{2}\right]=\mathbb{E}_{\mathcal{Y}_{T}}\left[\left\|\hat{x}_{t}^{s}\right\|^{2}\right]+\mathbb{E}_{\mathcal{Y}_{T}}\left[\left\|\hat{x}_{t}^{o}\right\|^{2}\right]$. Using Lemmas 10 and 12 we conclude that $\mathbb{E}_{\mathcal{Y}_{T}}\left[\left\|\hat{x}_{t}\right\|^{2}\right] \leqslant \gamma_{s}+\gamma_{o}$, for all $t \geqslant T$, and therefore, $\mathbb{E}_{\mathcal{Y}_{T}}\left[\left\|x_{t}\right\|^{2}\right] \leqslant 2 \rho+2\left(\gamma_{s}+\gamma_{o}\right)$, for all $t \geqslant T$. Since the sequence $\left(x_{t}\right)_{t \in \mathbb{N}}$ in (1a) is generated through the addition of independent mean-square bounded random variables and a bounded control input, and since $T<\infty$, it follows that there exists a constant $\gamma>0$ such that $\mathbb{E}_{\mathcal{Y}_{0}}\left[\left\|x_{t}\right\|^{2}\right] \leqslant \gamma$, for all $t \in \mathbb{N}$, establishing the claim (ii) of Theorem 5 .

PROOF. [Proof of Corollary 6] The proof of Corollary 6 follows exactly the same reasoning as in the proof of Theorem 5, except for the constraints in (34) and (35). Rewriting the constraints (30) and (31) as (34) and (35), respectively, can be done similarly to the way we rewrote the cost (6) in Theorem (5). It remains to show the upper bounds $\alpha^{*}$ and $\beta^{*}$ in (33). For the constraint (30) we have the upper-bound $\mathbb{E}_{\mathcal{Y}_{t}}\left[\left\|X_{t}\right\|_{\mathcal{S}}^{2}+\mathcal{L}^{\top} X_{t}\right]=\mathbb{E}_{\mathcal{Y}_{t}}\left[\left\|\mathcal{A} x_{t}+\mathcal{B} U_{t}+\mathcal{D} W_{t}\right\|_{\mathcal{S}}^{2}\right.$ $\left.+\mathcal{L}^{\top}\left(\mathcal{A} x_{t}+\mathcal{B} U_{t}+\mathcal{D} W_{t}\right)\right] \leqslant 3 \mathbb{E}_{\mathcal{Y}_{t}}\left[\left\|\mathcal{A} x_{t}\right\|_{\mathcal{S}}^{2}+\left\|\mathcal{B} U_{t}\right\|_{\mathcal{S}}^{2}\right.$ $\left.+\left\|\mathcal{D} W_{t}\right\|_{\mathcal{S}}^{2}\right]+\mathcal{L}^{\top} \mathcal{A} \hat{x}_{t}+\left|\mathcal{L}^{\top} \mathcal{B} U_{t}\right| \leqslant \mathcal{L}^{\top} \mathcal{A} \hat{x}_{t}$ $+3 N m \sigma_{\max }\left(\mathcal{B}^{\top} \mathcal{S B}\right) U_{\max }^{2}+\left\|\mathcal{L}^{\top} \mathcal{B}\right\|_{1} U_{\max }$ $+3 \operatorname{tr}\left(\mathcal{A}^{\top} \mathcal{S} \mathcal{A} \mathbb{E}_{\mathcal{Y}_{t}}\left[x_{t} x_{t}^{\top}\right]+\mathcal{D}^{\top} \mathcal{S D} \Sigma_{w}\right)$, where the first inequality follows from the fact that $(a+b+c)^{2} \leqslant$ $3\left(a^{2}+b^{2}+c^{2}\right)$, for any $a, b, c>0$, and the noise being zero-mean; the second inequality follows from applying norm bounds between the 2 and $\infty$-norms and Hölder's inequality. As for the constraint (31), we have the bound $\mathbb{E}_{\mathcal{Y}_{t}}\left[U_{t}^{\top} \tilde{\mathcal{S}} U_{t}\right] \leqslant \sigma_{\max }(\tilde{\mathcal{S}})\left\|U_{t}\right\|^{2} \leqslant$ $N m \sigma_{\max }(\tilde{\mathcal{S}})\left\|U_{t}\right\|_{\infty}^{2} \leqslant N m \sigma_{\max }(\tilde{\mathcal{S}}) U_{\max }^{2}$. 